\title{
Assessment of thermo-hydraulic performance of inward dimpled tubes with variation in angular orientations
}

\section{Rizwan Sabir, Muhammad Mahabat Khan, Nadeem Ahmed Sheikh, Inam Ul-Ahad, Dermot Brabazon}

aDepartment of Mechanical Engineering, Capital University of Sciences and Technology, Islamabad, Pakistan

${ }^{\mathrm{b}}$ Department of Mechanical Engineering, International Islamic University, Islamabad, Pakistan

'I-Form Advanced Manufacturing Research Centre, Dublin City University, Dublin, Ireland

Corresponding author: andre.mussatto2@mail.dcu.ie

\begin{abstract}
This paper presents a numerical investigation and assessment of thermal and hydraulic performance of dimpled tubes of varying topologies at constant heat flux of and Reynolds numbers ranging from 2300 to 15,000 The performance of the tubes consisting of conical, spherical and ellipsoidal dimples with equivalent flow volumes were compared using steady state Reynolds Averaged Navier Stokes simulations. The ellipsoidal dimples, in comparison to other dimple shapes, demonstrated large increment in heat transfer rate. The variation in the orientation of the ellipsoidal dimples was examined to further improve thermal and hydraulic performances of the tube. A $45^{\circ}$ inclination angle of ellipsoidal dimple, from its major axis, increased the thermo-hydraulic performance by $58.1 \%$ and $20.2 \%$ in comparison to smooth tube and $0^{\circ}$ ellipsoidal dimpled tube, respectively. Furthermore, Large Eddy Simulations (LES) were carried out to investigate the role geometrical assistance to fluid flow and heat transfer enhancement for the $45^{\circ}$ and $90^{\circ}$ ellipsoidal dimpled tubes. LES results revealed a flow channel of connected zones of wakes which maximized fluid-surface contact and therefore enhanced the thermal performance of the tube. In addition, correlations for Nusselt number and friction factor for all angular topologies of ellipsoidal dimpled tube have been proposed.
\end{abstract}

\section{Keywords}

Heat transfer enhancement, Nusselt number, Large Eddy Simulation, Turbulent flow, Enhanced tubes

To cite this article: Rizwan Sabir, Muhammad Mahabat Khan, Nadeem Ahmed Sheikh, Inam Ul-Ahad, Dermot Brabazon, Assessment of thermo-hydraulic performance of inward dimpled tubes with variation in angular orientations, Applied Thermal Engineering, Volume 170, 2020, 115040. 


\section{Introduction}

The most widely used component in many heat transfer devices is a tube which transports the fluid to either gain or dissipate heat. Thermo-hydraulics of the transport phenomena involved in the tube flow has been extensively studied, both experimentally and numerically. The compactness of thermal devices along with thermo-hydraulic performance enhancement demands more efficient, innovative, economical and reliable solutions. In this regard, different heat transfer enhancement methods in the tubes have been employed in the past. Heat transfer enhancement by increasing surface roughness inside the tubes was achieved by Bergles et al. [1]. Boundary layer of the flow inside the tube was disturbed by the rough surface which increased fluid-surface interaction and resultant heat transfer. Helical ribroughened tubes were also used to increase the heat transfer rates [2]. Similarly, the use of ribturbulators have also been found to enhance heat transfer rate [3]. The effects of rib angles, ribs spacing, channel aspect ratio, rib height on the heat transfer rate of the tube, at high Reynolds numbers were explored. Garcia et al. [4] performed an experimental study by incorporating the helical-wire-coils inside the tubes to improve thermo-hydraulic performance of the tubes for a wide range of Reynolds numbers and Prandtl numbers. In continuation to a previous study, Garcia et al. [5] compared the thermo-hydraulic performance of helical-wire-coils and dimpled tubes. It was demonstrated that the performance of dimpled tubes was much superior to the wire-coils, especially for turbulent flows. Dimples increased the surface area and disturbed the velocity boundary layer of the flow which in return increased the heat transfer rate but at the cost of enhanced friction losses [6], [7].

Experimental study of horizontal dimpled tubes with R-134a as a working fluid was performed by Aroonrat et al. [8]. In comparison to smooth tube, they managed to increase the heat transfer coefficient by 1.7 times by using dimpled tube. Maithani et al. [9] achieved a significant increase in the performance of heat exchanger by introducing dimpled tubes. The performance assessment of trapezoidal dimpled tube was performed by Dagdevir et al. [10]. It was concluded that trapezoidal dimpled tubes improved heat transfer rate considerably in comparison to smooth tubes. The effect of depth of the dimples of enhanced tube was investigated by Cheraghi et al. [11]. They demonstrated that the heat transfer enhancement of deep dimpled tubes was linked to axial swirling of flow caused by the vortices in the wake of dimples.

The effect of different patterns of conical dimples on the heat transfer enhancement was investigated by Fan et al. [12] using three dimensional numerical simulations. The inline dimple pattern showed higher heat transfer rate as compared to staggered dimples. Li et al. [13] performed three-dimensional steady state numerical simulations of three different tubes with conical, spherical and ellipsoidal dimples. The study recommended inline ellipsoidal dimples in comparison to conical and spherical dimples for heat transfer enhancement in tubes. Liang et al. [14] investigated thermal hydraulic performance of ellipsoidal dimpled tubes. It was observed that the dimple arrangement significantly affected the heat transfer rate. For $\operatorname{Re}<104$, the ellipsoidal dimples with major axis aligned to the flow direction (i.e., ellipsoidal $0^{\circ}$ ) resulted in highest heat transfer rate. However, for $\operatorname{Re}>104$, the ellipsoidal dimple with $60^{\circ}$ inclination of the major axis with respect to the flow direction (i.e., ellipsoidal $60^{\circ}$ ) demonstrated highest heat transfer rate. The heat transfer enhancement in a tube with combination of ellipsoidal dimples in vertical and horizontal directions, with respect to flow direction, was studied by Xie et al. [15]. It was observed that the heat transfer was increased by increasing the depth and axis ratio of the dimples. The performance of mini-channels with spherical dimples were compared to channels with cylindrical grooves and fins by Bi et al. [16]. Chen et al. [17] experimentally studied the modified tubes with spherical dimples for incompressible flow. It was observed that heat transfer enhanced from $25 \%$ to $137 \%$ at constant Re, and from $15 \%$ to $84 \%$ at constant pumping power. Thermal performance enhancement of dimpled tubes was also demonstrated by [18], [19].

More recently, thermal and hydraulic performance of a teardrop dimpled tube was compared to spherical and ellipsoidal $0^{\circ}$ dimpled tubes by Xie et al. [20]. Heat transfer rate of tear-drop dimpled 
tube showed higher heat transfer rate than the ellipsoidal and spherical dimpled tubes with lower friction factors. Thermo-hydraulic performance enhancement of the teardrop dimpled tube was attributed to the flow separation behind the dimple and smooth mixing in the wake. Since the effect of wake structure seems to the prime factor influencing the heat transfer enhancements, the study of performance of the teardrop dimpled tube was not compared with different orientations of the ellipsoidal dimple. These orientations can play crucial role in the heat transfer enhancement by altering the wake structure. It was concluded that the dimpled surfaces provided the highest heat transfer rate in comparison to cylindrical grooves and fins.

It is important to note that the total tube volume for each dimple topology must be considered while comparing different geometrical features. Since the thermo-hydraulic performances are generally compared for different topological variations in tubes; the overall flow volume is expected to be kept constant for comparison. One may argue that the overall Reynolds number should be kept the same, however it is pertinent to mention that the Reynolds number is generally calculated using the hydraulic diameter, normally taken as base tube diameter, as the characteristic length. With the introduction of dimples, the local Reynolds number will be altered which will in return alter the flow physics and friction factor of the tube. However, a like-for-like case for comparison can only be expected if the tubes deliver or offer same flow volumes. As noted in the literature, in most previous studies such consideration was not ensured and consequently, the surface area for each dimpled tube was effectively different. Therefore, the role of dimple shape especially when subjected to a comparison considering thermo-hydraulic performance aspect remains an open question.

In this study, the flow and heat transfer characteristics in dimpled tubes were investigated using numerical simulations. In order to ensure that the available volume for flow of incompressible fluid remained same, the dimple volumes were kept constant. Therefore, equal surface area of the dimples for each shape and orientation was ensured. The influence of the dimple geometry and its arrangement patterns on the heat transfer and flow characteristics were studied for a wide range of Reynolds numbers. Through a parametric study, identification of the optimum dimple geometry as well as arrangement configuration were investigated using Reynolds Averaged Navier Stokes (RANS) under steady flow conditions. The flow physics which resulted in increased thermo-hydraulic performance of the optimum configuration was further investigated using Large Eddy Simulation (LES) to highlight the transport mechanism of heat and momentum. LES results provided insights on the unsteady dimpled tube flow with high resolution of the wake, elaborating the role of orientation of dimples and their spacing in terms of wake-dimple interaction.

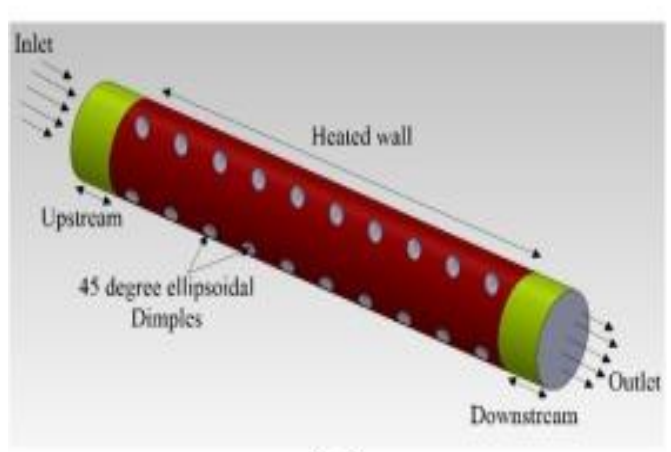

(a)

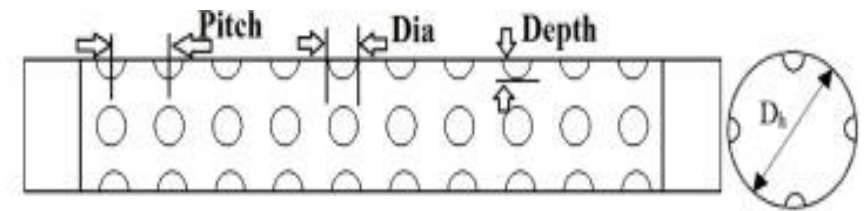

(b)

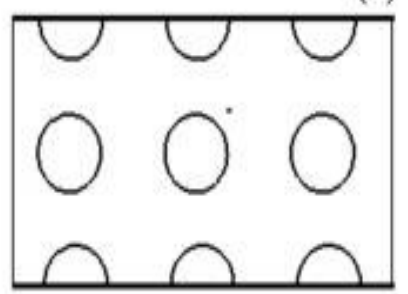

(d) (c)

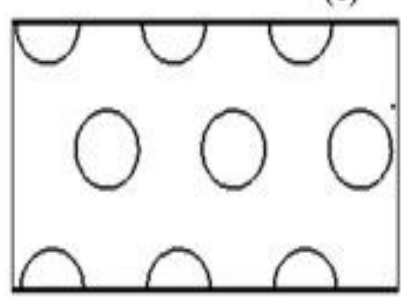

(e)

Fig. 1. (a) Geometry of the ellipsoidal dimpled tube (b) geometric properties of dimple (c) cross-section of dimpled tube (d) inline configuration (e) staggered configuration. 


\section{Computational domain}

\subsection{Geometry}

The computational domain was a tube of internal diameter $\mathrm{Dh}$ with dimple diameter $\mathrm{Dp}$, depth $\mathrm{H}$ and pitch P, as shown in Fig. 1(a), (b) and (c). The length of the tube was $120 \mathrm{~mm}$; however, the effective enhanced tube length was selected to be $100 \mathrm{~mm}$ in order to eliminate any spurious effects of inlet and outlet boundary conditions. The inward facing dimples in the tube were positioned in the in-line and staggered arrangements, as presented in Fig. 1(d and e). The dimples in the inline configuration were in-phased while in the staggered configuration dimples were out of phase. The number of dimples in circumferential direction is referred in this study as "Star". Ten dimples were used in the axial direction for all configurations. The dimples shape along with other geometrical parameters are presented in Table 1. Three different shapes of dimples consisting of conical, spherical and ellipsoidal profiles were used which are shown in Fig. 2.

Table 1. Parameters of all tubes used for validation.

\begin{tabular}{|c|c|c|c|c|c|c|c|}
\hline $\begin{array}{l}\text { Sr. } \\
\text { No }\end{array}$ & $\begin{array}{l}\text { Internal Diameter of } \\
\text { tube }\left(D_{h}\right)(\mathrm{mm})\end{array}$ & $\begin{array}{l}\text { Dimple } \\
\text { Profile }\end{array}$ & $\begin{array}{l}\text { Depth }(\mathrm{H}) \\
(\mathrm{mm})\end{array}$ & $\begin{array}{l}\text { Pitch }(\mathrm{P}) \\
(\mathrm{mm})\end{array}$ & $\begin{array}{l}\text { Dimple } \\
\text { Diameter }\left(\mathrm{D}_{\mathrm{p}}\right) \\
(\mathrm{mm})\end{array}$ & Star & Configuration \\
\hline $\begin{array}{l}\text { Tube } \\
1\end{array}$ & 17.272 & conical & 1.2 & 10 & 5 & 6 & staggered \\
\hline $\begin{array}{l}\text { Tube } \\
2\end{array}$ & 17.272 & conical & 1.2 & 10 & 5 & 6 & Inline \\
\hline $\begin{array}{l}\text { Tube } \\
3\end{array}$ & 17.272 & conical & 1.2 & 10 & 5 & 4 & Inline \\
\hline $\begin{array}{l}\text { Tube } \\
4\end{array}$ & 17.272 & spherical & 2.5 & 10 & 5 & 4 & Inline \\
\hline $\begin{array}{l}\text { Tube } \\
5\end{array}$ & 17.272 & $\begin{array}{l}\text { ellipsoidal } \\
\left(0^{\circ}\right)\end{array}$ & 1.5 & 10 & 5 & 4 & Inline \\
\hline
\end{tabular}

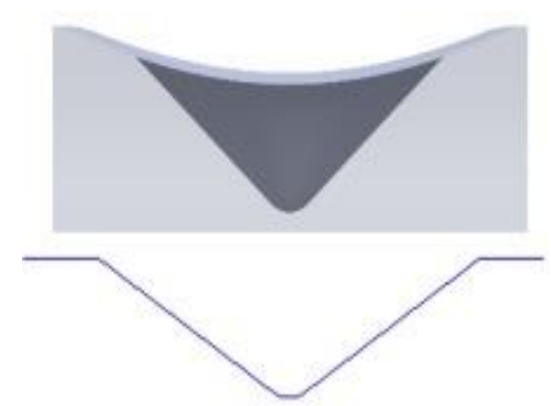

(a)

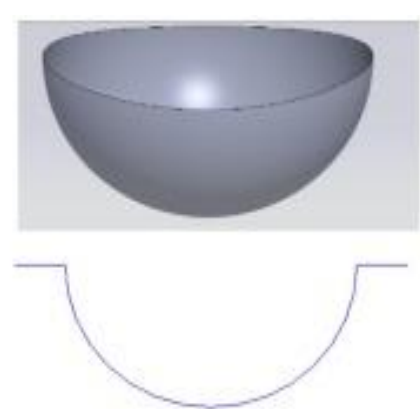

(b)

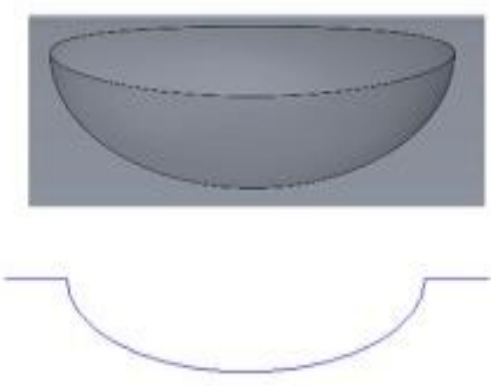

(c)

Fig. 2. Shapes of dimples (a) conical (b) spherical (c) ellipsoidal. 


\subsection{Boundary conditions}

The flow at the inlet of the channel was considered to be fully developed. A turbulent inlet velocity profile, representing the fully developed flow using 1/7th power law [21], was imposed as the inlet boundary condition. The velocity profile at the inlet of the tube is presented in Eq. (1)

$$
\frac{U}{U_{\mathrm{c}}}=\left(1-\frac{r}{D_{h}}\right)^{1 / 7}
$$

where $r$ denotes the radial distance, $D_{h}$ is the hydraulic diameter. At the outlet, pressure outlet boundary condition was used which considers zero-gauge pressure at outlet and at the walls, no slip condition was used. The working fluid was water with constant fluid properties is used. The density of water is $994.57 \mathrm{~kg} / \mathrm{m}^{3}$, dynamic viscosity of $7.5407 \times 10^{-4} \mathrm{~kg} / \mathrm{m} \cdot \mathrm{s}$, thermal conductivity of $0.62215 \mathrm{~W} / \mathrm{m} \cdot \mathrm{K}$ with Prandtl number of 4.8734 . The operating temperature range in this work was small therefore these properties were considered to be constant.

Generally, the surface of the tubes can be heated by maintaining three different types of thermal boundary conditions: (i) uniform wall temperature circumferentially and uniform heat flux axially, (ii) uniform wall heat flux in both axial and circumferential direction, and (iii) uniform wall temperature. However, according to [13], the choice of thermal boundary conditions does not effect thermal and hydraulic trends of the enhanced tubes. Therefore, in the current study, a uniform wall heat flux in both axial and circumferential direction was maintained.

The value of heat flux was set as 10, which was uniformly applied along the length of the tube as shown in Fig. 1(a). This heat flux value was selected as the reference value based on the experimental work of $\mathrm{Li}$ et al. [22]. The adiabatic condition was applied at the upstream and downstream portion of the dimpled tube as shown in Fig. 1(a). The collective information of boundary conditions is given in Table 2.

Table 2. Boundary condition (BC) for the tube analysis and modelling.

\begin{tabular}{ll} 
Inlet & Velocity inlet BC \\
\hline Inlet (thermal) & $305.5 \mathrm{~K}$ \\
Outlet & Pressure outlet (0-gauge pressure) \\
Wall (friction) & No slip BC \\
Wall (thermal) & Constant heat flux $10 \mathrm{~kW} / \mathrm{m}^{2}$ \\
Up and downstream & Adiabatic condition \\
Working fluid & Water, with constant properties
\end{tabular}




\section{Mathematical formulation}

\subsection{Governing equation}

The flow is governed by incompressible mass, momentum and energy conservation equations. The vector form of these equations are presented as Eqs. (2), (3), (4), respectively.

$$
\begin{aligned}
& \frac{\partial\left(u_{j}\right)}{\partial x_{j}}=0 \\
& \frac{\partial}{\partial t}\left(\rho u_{j}\right)+\frac{\partial}{\partial x_{j}}\left(\rho u_{i} u_{j}\right)=-\frac{\partial p}{\partial x_{i}}+\frac{\partial}{\partial x_{j}}\left[\left(\mu+\mu_{t}\right)\left(\frac{\partial u_{i}}{\partial x_{i}}+\frac{\partial u_{j}}{\partial x_{i}}\right)\right] \\
& \frac{\partial T}{\partial t}+\frac{\partial u_{i} T}{\partial x_{i}}=\frac{\partial}{\partial x_{j}}\left[\left(\frac{\mu}{P r}+\frac{\mu_{t}}{P r_{t}}\right) \frac{\partial T}{\partial x_{j}}\right]
\end{aligned}
$$

where $\mathrm{T}, u, \mu, \rho$ are temperature, velocity, dynamic viscosity and density respectively. The turbulent viscosity and turbulent Prandtl number are represented by and $\mu_{t}$ and $\operatorname{Pr}_{t}$, respectively.

\subsection{Turbulence models}

The above governing conservation equations of mass, momentum and energy were ensemble averaged for the steady state simulations and filtered for unsteady simulations. The steady state simulations were performed by Reynolds Averaged Navier Stokes (RANS) formulation while unsteady simulations were performed by Large Eddy Simulation (LES) framework.

\subsubsection{Transport equations for shear stress transport (SST) $k-\omega$ model}

The turbulence properties in RANS were computed by incorporating closure using SST $k-\omega$ model. This model uses standard $k-\omega$ model in near wall regime while in free stream region, it uses $k-\varepsilon$ model. SST $k-\omega$ model showed good results in confined flows where near wall effects were important [13]. The governing equations of SST $k-\omega$ model [23] have been presented in Eqs. (5), (6)

$$
\begin{aligned}
& \frac{\partial}{\partial t}(\rho k)+\frac{\partial}{\partial x_{i}}\left(\rho k u_{i}\right)=\frac{\partial}{\partial x_{j}}\left(\Gamma_{k} \frac{\partial k}{\partial x_{j}}\right)+\widehat{G}_{k}-Y_{k}+S_{k} \\
& \frac{\partial}{\partial t}(\rho \omega)+\frac{\partial}{\partial x_{i}}\left(\rho \omega u_{i}\right)=\frac{\partial}{\partial x_{j}}\left(\Gamma_{\omega} \frac{\partial \omega}{\partial x_{j}}\right)+\widehat{G}_{\omega}-Y_{\omega}+D_{\omega}+S_{\omega}
\end{aligned}
$$


where $k$ and $\omega$ are the turbulence kinetic energy and specific

dissipation rate. $\widehat{G}_{k}$ is the turbulence kinetic energy, $\widehat{G}_{\omega}$ is

generation of $\omega$, while $\Gamma_{k}$ and $\Gamma_{\omega}$ denote the effective

diffusivity of $\mathrm{k}$ and $\boldsymbol{\omega}$ respectively. Furthermore, $\mathrm{Y}_{k}$ and $Y_{\omega}$

represent the turbulence dissipation of $\mathrm{k}$ and $\boldsymbol{\omega}$ respectively.

$\mathrm{D}_{\omega}$ is the cross-diffusion, $\mathrm{S}_{k}$ and $S_{\omega}$ are source terms.

The instantaneous behavior of the flow was modeled using Large Eddy Simulations (LES). The dimples on the surface of the tube can generate secondary flow which consist of different scales of eddies. LES resolves large-scale eddies and models small-scale universal eddies. The accuracy of LES depends on the size of small eddies that are selected through eddy scale separation process. The scale separation of the eddies is performed by filtering out governing equations

Eqs. (1), (2), (3) by using a cutoff top hat filter $\left(\Delta_{\text {cell }}\right)$. The filter size was equal to the cube root of size of computational cell [23], which yielded $\Delta_{\text {cell }}=19.4 \mu \mathrm{m}$ for the current case. The turbulent viscosity $\left(\mu_{t}\right)$ used to account for the turbulent effect of small-scale eddies, was modeled using Standard Smagorinsky model [24], [25] presented in Eq. (7).

$\mu_{t}=\rho\left(C_{s} \Delta\right)^{2} \sqrt{2 \bar{S}_{i j} \bar{S}_{i j}}$

where $\bar{S}_{i j}$ is rate of strain tensor for resolved scales and $C_{s}$ is the Smagorinsky constant and its value was selected to be 0.1 .

\subsection{Parameter definitions}

Reynolds numbers is a dimensionless number, defined as the ratio of inertial to viscous forces, has been presented in Eq. (8).

$R e=\frac{\rho U D_{h}}{\mu}$

where $U$ is velocity and $D_{h}$ is the hydraulic diameter of the tube which is geometric diameter of the tube.

Convective heat transfer coefficient $\left(h_{i}\right)$ is the ratio heat flux to temperature difference between wall and the fluid. The relationship of $h_{i}$ has been presented in the Eq. (9).

$h_{i}=\frac{q "}{T_{\text {wadl }}-T_{\infty}}$

where $q$ " indicates the heat flux, $T_{\infty}$ mean temperature i.e. mass weighted average temperature of the fluid inside the tube, and $T_{\text {wall }}$ is the wall local temperature.

The ratio of convective heat transfer to the conductive heat transfer of a fluid is represented by a non-dimensional Nusselt number $(\mathrm{Nu})$, which was locally determined [23] as: 
$N u_{i}=\frac{h_{i} D_{h}}{k}$

where $k$ is thermal conductivity of the fluid.

Darcy friction factor was used to determine hydraulic loss of the tube flow, as shown in Eq. (11):

$f r=\frac{2 \Delta p D_{h}}{\rho U^{2} L}$

where $\Delta p$ represents the pressure difference between inlet and outlet of a tube.

\subsection{Numerical method}

Finite volume method was used to discretize the governing equations which were solved using the steady state implicit format in ANSYS Fluent $\AA$ [23]. Pressure velocity coupling was solved by pressure based coupled algorithm which solved continuity and momentum equations together. The coupled algorithm was more robust and gave faster convergence than the traditional segregated algorithms [23]. The gradients in diffusion terms of governing equations were solved by least squares cell-based method. The pressure gradient was approximated using second order interpolation scheme while second order upwind scheme was used for momentum and energy. The convergence criterion was $10^{9}$ for the residuals of continuity, momentum and energy.

\subsection{Grid independence study}

The domain discretization was performed by generating a structured mesh with refinement at near plane wall and dimple surface, as presented in Fig. 3. A high-quality mesh around the dimples was imperative since the flow separation, attachment, vortex formation and flow mixing take place in the vicinity of the dimple. Therefore, additional care was taken while generating the mesh around the dimples. A thorough mesh independence study was conducted and results for the case of tube 3 at $\mathrm{Re}=5000$ and $\mathrm{Re}=9000$ are presented below. The resultant $\mathrm{Nu}$ and fr were presented in Table 3 for different mesh sizes for the selected cases. The number of cells, for different mesh sizes, were varied from 0.28 million (Coarse mesh) to 6.5 million (Fine Mesh). The percentage difference in $\mathrm{Nu}$ and $\mathrm{fr}$ increased as the mesh size was reducing, however, it became constant on a 2.0 million mesh size. In order to ensure that the mesh dependent variations were taken care of, a 3.0 million mesh was selected for all computational analyses presented in this paper. Moreover, the accuracy of the solution of turbulent flow depends on the resolution of the mesh in boundary layer region. In the selected mesh size of 3.0 million, the resultant $y^{+} \sim 0.51$ was noted which ensured that the mesh size was adequate to resolve the near wall effects.

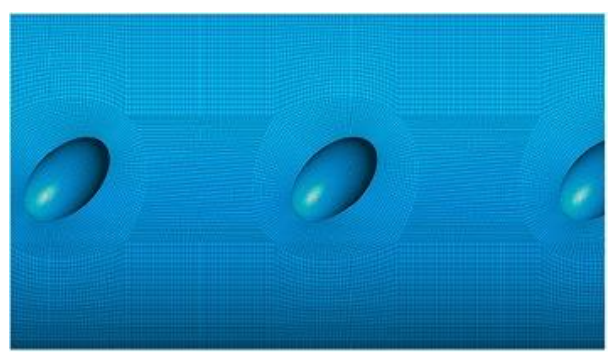

Fig. 3. Structured grid of ellipsoidal dimpled tube. 
Table 3. Grid independence study of conical staggered enhanced tube (tube 3).

\begin{tabular}{lllll} 
Grid (Million) & \multicolumn{3}{l}{$R e=5000$} & $R e=9000$ \\
\cline { 2 - 5 } & $\mathrm{Nu}$ & $f r$ & $N u$ & $f r$ \\
\hline 0.28 & 74.8 & 0.0820 & 112.0275 & 0.0761 \\
1 & 69.36 & 0.0780 & 112.2322 & 0.0701 \\
2 & 68.33 & 0.07815 & 111.9493 & 0.0679 \\
3 & 68.31 & 0.0783 & 111.9873 & 0.06592 \\
6.5 & 68.30 & 0.07835 & 111.9879 & 0.0650
\end{tabular}

\section{Results and discussion}

\subsection{Validation}

The validation of numerical methodology was performed by comparing the results of smooth tubes with empirical correlations and the dimpled tubes with experimental results from literature. Typically, the most appropriate correlation for assessing the heat transfer of smooth tube would be the Gnielinski correlation [26]. However, the tube considered in current study is a short tube and therefore, suitable empirical correlations to compare the numerical results is the modified version of Gnielinski correlation of Taler [27], as shown in Eq. (12). The friction factor was compared to Filonienko correlation [28] for smooth tube in Eq. (13). Although one may argue that Filonienko correlation of $f r$ was proposed for a long pipe however, for the sake of completeness this correlation was used for comparison.

$$
\begin{aligned}
& N u=\frac{(f r / 8)(R e-1000) P r}{1+12.7\left(\frac{f r}{8}\right)^{\frac{1}{2}}\left(\operatorname{Pr}^{\frac{2}{3}}-1\right)}\left[1+\left(\frac{D_{h}}{L}\right)^{2 / 3}\right] \\
& f r=(1.82 \log \mathrm{Re}-1.64)^{-2} \\
& \text { The Eq. (12) is valid for a large range of } \operatorname{Pr} ; 0.5 \leq P r \leq 2000 \text {. } \\
& \text { The Eqs. (12), (13) are valid for } 3000 \leq R e \leq 5 \times 10^{6} \text {. } \\
& \text { Fig. 4(a) and (b) shows comparison between numerical results } \\
& \text { and experimental correlations of smooth tubes. Typically, in } \\
& \text { smooth tubes, } N u \text { is directly proportional to the Re while } f r \\
& \text { is inversely proportional to the Re. This trend could be } \\
& \text { observed in Fig. } 4 \text { which shows that the numerical } \\
& \text { simulations also predict increase in } N u \text { with increasing } R e \\
& \text { and a decrease in } f r \text { with increase in } R e \text {. The percentage } \\
& \text { difference in } N u \text { and } f r \text { is well within the range of } 30 \% \text { as } \\
& \text { expected for pipes with short length. The maximum } \\
& \text { difference is observed at the flow transition region. }
\end{aligned}
$$

The numerical results were also validated for the enhanced tubes and here some of the results with conical dimples are presented. The $\mathrm{Nu}$ and fr of tube 1 and tube 2 (referred to Table 01) were compared with experimental results of $\mathrm{Li}$ et al. [22]. The results from the present numerical study show a reasonably accurate comparison to the experimental and numerical results. It can be observed from Fig. $5(\mathrm{a}, \mathrm{b})$ that $\mathrm{Nu}$ increased and fr decreased for both tube 1 and tube 2 with increasing. A maximum deviation of $19.61 \%$ was observed for $\mathrm{Nu}$ and $18.34 \%$ for fr predictions. It is important to mention that the turbulence models in RANS are known to over predict the hydraulic losses and heat transfer 
coefficient in the transition region [12], [13]. Therefore, the discrepancies between the experimental and numerical results were expected in the transition region and reasonably matched well with the previously reported trends. It can be noted that the maximum difference between the experimental and numerical results was observed in the transition Re region. However, as the flow became fully turbulent, the turbulence closure model improved the accuracy of the results by reducing the percentage difference to less than $2 \%$ for $\mathrm{Nu}$ and $10 \%$ for fr .

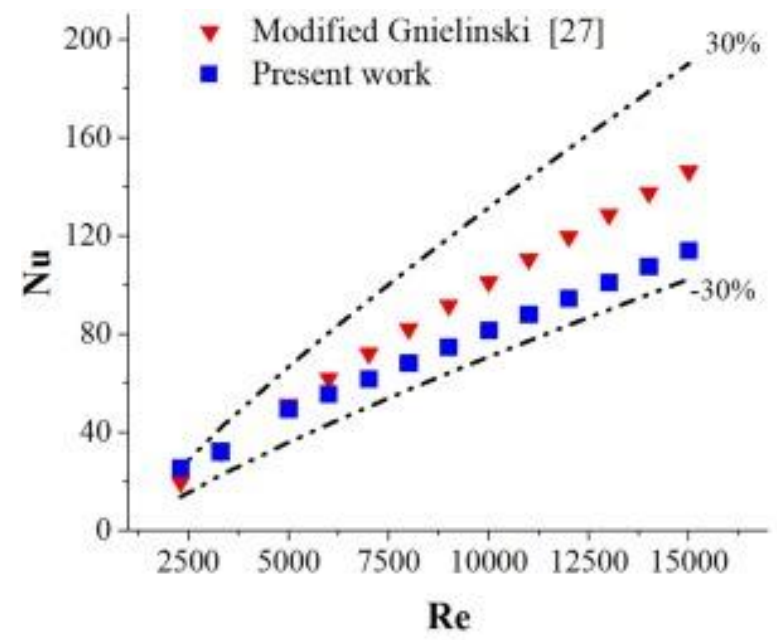

(a)

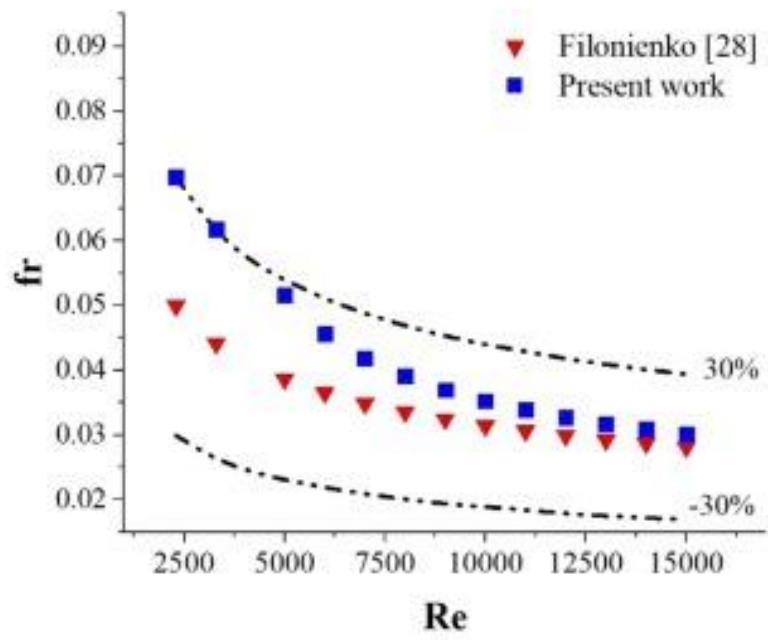

(b)

Fig. 4. Comparison of numerical results with empirical correlations (a) $\mathrm{Nu}$ and (b) fr.

The numerical results were also validated for the enhanced tubes and here some of the results with conical dimples are presented. The $N u$ and $f r$ of tube 1 and tube 2 (referred to Table 01) were compared with experimental results of Li et al. [22]. The results from the present numerical study show a reasonably accurate comparison to the experimental and numerical results. It can be observed from Fig. 5(a, b) that $N u$ increased and $f r$ decreased for both tube 1 and tube 2 with increasing Re. A maximum deviation of $19.61 \%$ was observed for $\mathrm{Nu}$ and $18.34 \%$ for $\mathrm{fr}$ predictions. It is important to mention that the turbulence models in RANS are known to over predict the hydraulic losses and heat transfer coefficient in the transition region [12], [13]. Therefore, the discrepancies between the experimental and numerical results were expected in the transition region and reasonably matched well with the previously reported trends. It can be noted that the maximum difference between the experimental and numerical results was observed in the transition $R e$ region. However, as the flow became fully turbulent, the turbulence closure model improved the accuracy of the results by reducing the percentage difference to less than $2 \%$ for $N u$ and $10 \%$ for $f r$. 


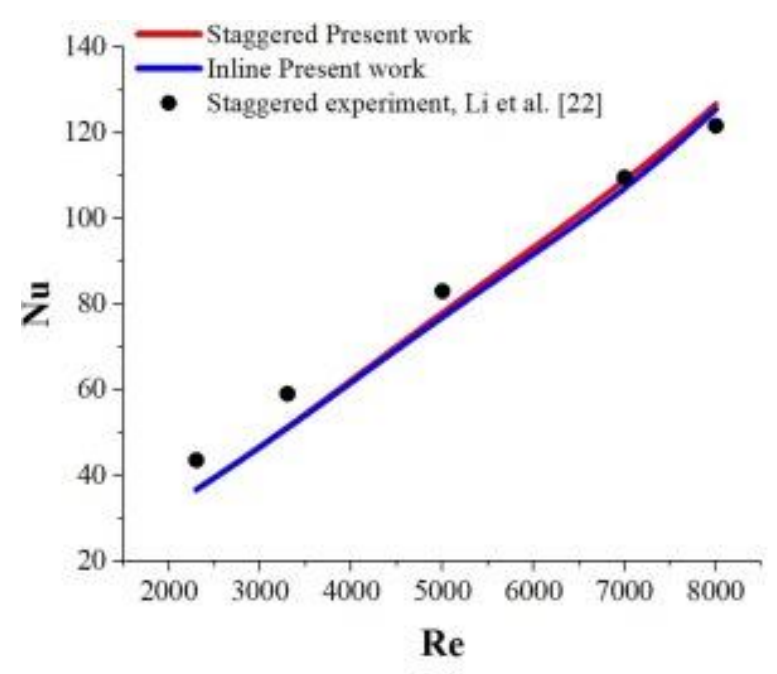

(a)

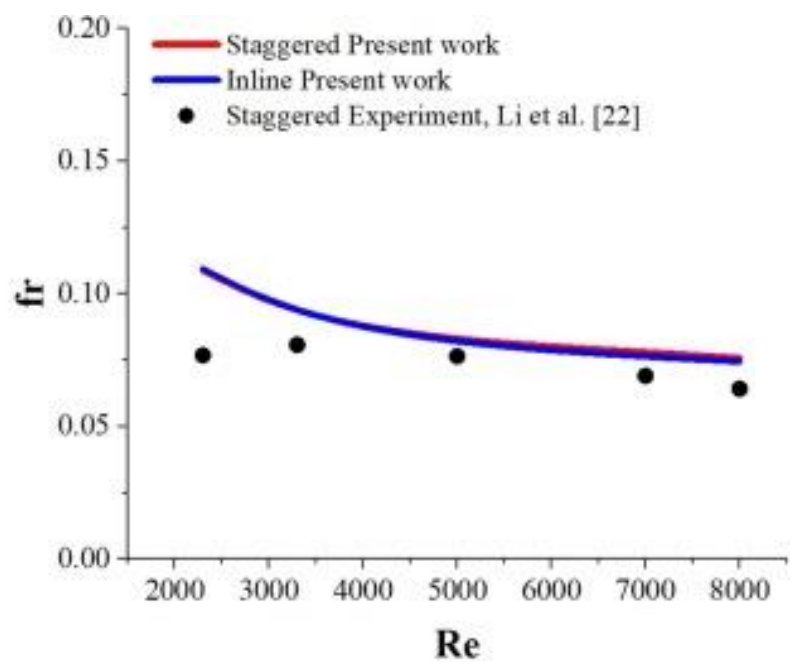

(b)

Fig. 4. Comparison of numerical results with empirical correlations (a) $\mathrm{Nu}$ and (b) fr.

The numerical results were also validated for the enhanced tubes and here some of the results with conical dimples are presented. The $N u$ and $f r$ of tube 1 and tube 2 (referred to Table 01) were compared with experimental results of $\mathrm{Li}$ et al. [22]. The results from the present numerical study show a reasonably accurate comparison to the experimental and numerical results. It can be observed from Fig. 5(a, b) that $N u$ increased and $f r$ decreased for both tube 1 and tube 2 with increasing Re. A maximum deviation of $19.61 \%$ was observed for $\mathrm{Nu}$ and $18.34 \%$ for $f r$ predictions. It is important to mention that the turbulence models in RANS are known to over predict the hydraulic losses and heat transfer coefficient in the transition region [12], [13]. Therefore, the discrepancies between the experimental and numerical results were expected in the transition region and reasonably matched well with the previously reported trends. It can be noted that the maximum difference between the experimental and numerical results was observed in the transition $R e$ region. However, as the flow became fully turbulent, the turbulence closure model improved the accuracy of the results by reducing the percentage difference to less than $2 \%$ for $\mathrm{Nu}$ and $10 \%$ for $\mathrm{fr}$. 


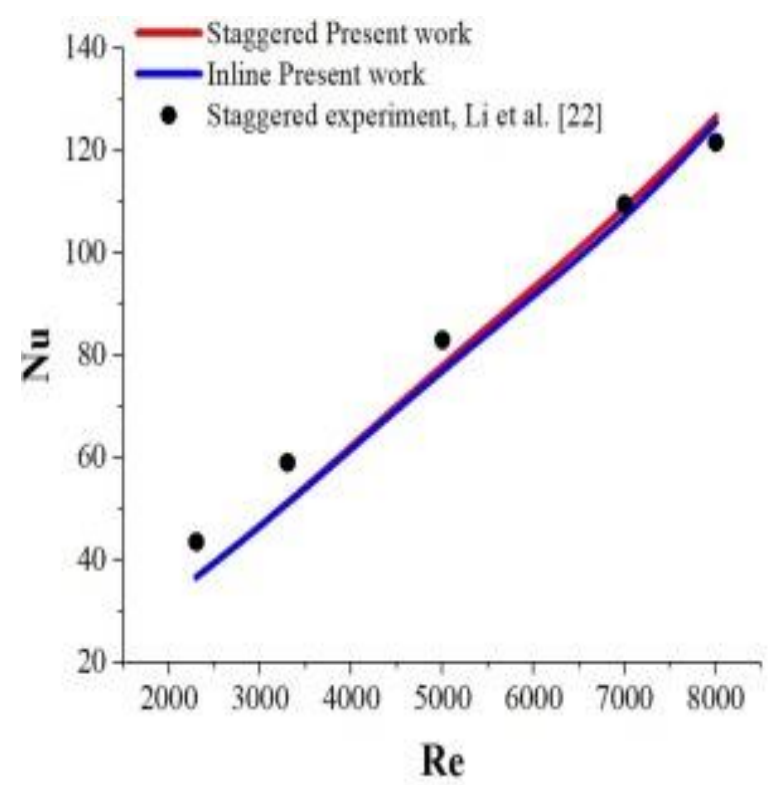

(a)

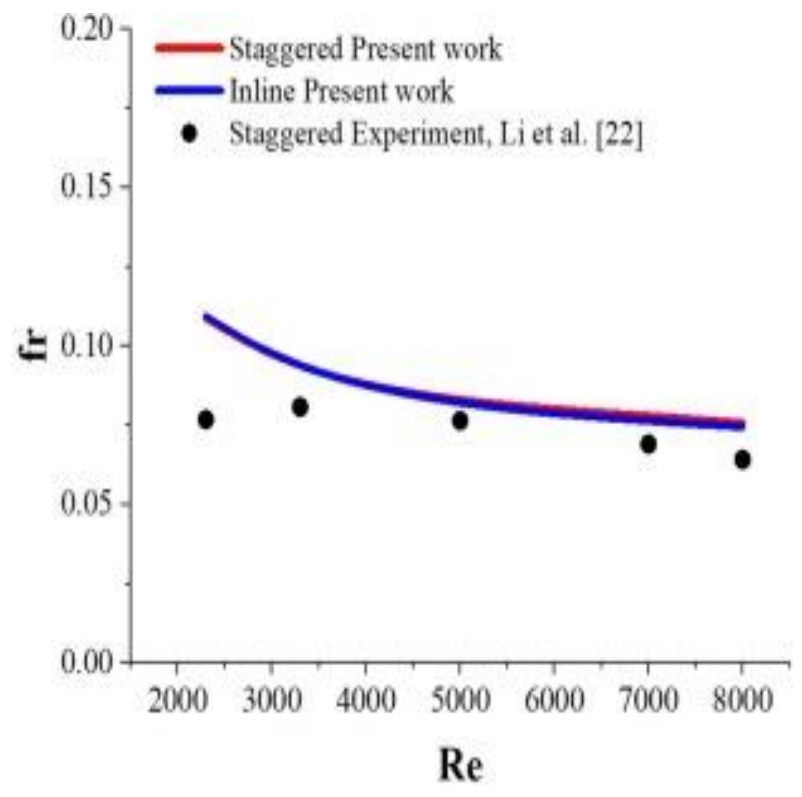

(b)

Fig. 5. Comparison of numerical results of tubes 1 and 2 with experimental results of Li et al. [22] (a) $\mathrm{Nu}$ and (b) fr.

The validations with the numerical results of $\mathrm{Li}$ et al. [13] were also performed for tube 3, tube 4 and tube 5. The $N u$ and $f r$ are compared in Fig. 6(a and b) for $2300 \leq R e \leq 15000$. It can be noted in Fig. 6(a) that the spherical dimple demonstrated highest heat transfer rate in comparison to ellipsoidal and conical dimpled tubes. Spherical dimpled tube showed $33.34 \%$ higher $N u$ than the conical dimpled tube and $27.7 \%$ higher than the ellipsoidal dimpled tube. The presented numerical results of $\mathrm{Nu}$ showed a very good agreement to the results of $\mathrm{Li}$ et al. [13]. It can also be noted from Fig. 6(b) that the sphere offered a relatively higher hydraulic loss in comparison to the other dimpled tubes. Again, a maximum deviation of $15.35 \%$ occurred in the transition Reynolds numbers range as compared to the results reported in the literature of $\mathrm{Li}$ et al. [13]. However, in the fully turbulent region a very good agreement was observed between the present study results and the results of literature [13]. 


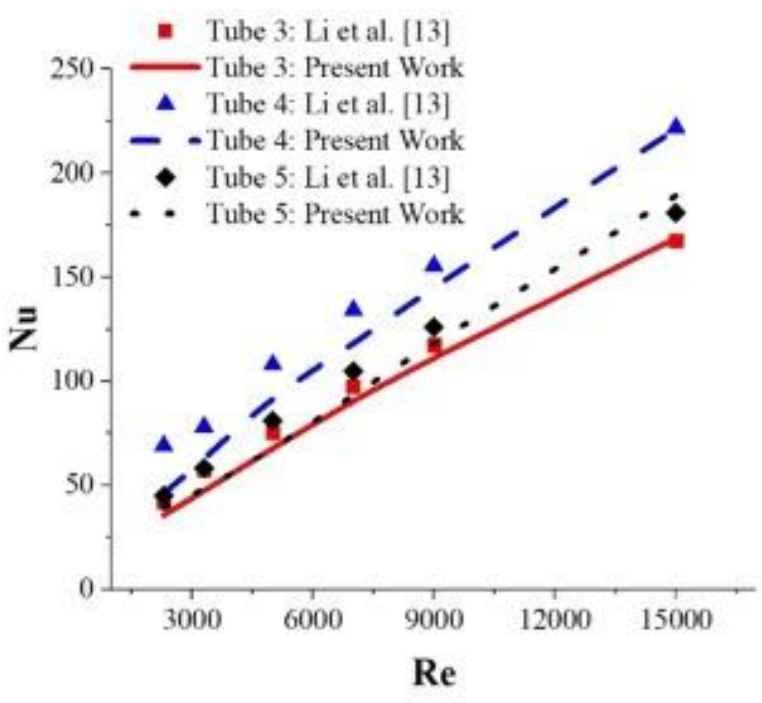

(a)

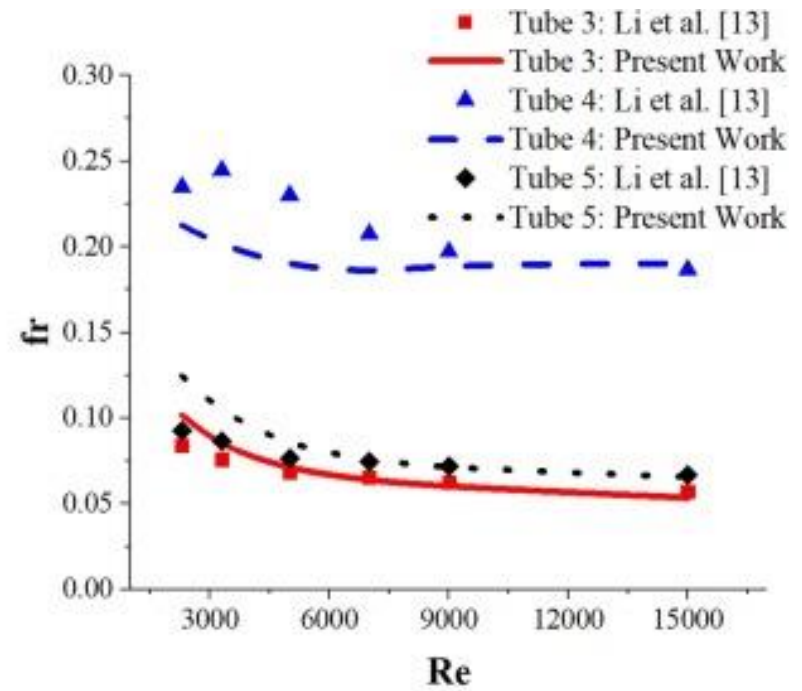

(b)

Fig. 6. Comparison between the numerical results of $\mathrm{Li}$ et al. [13] and the numerical results of present work for the tubes (3-5) (a) $\mathrm{Nu}$ and (b) fr.

\subsection{Thermal and hydraulic characterization of enhanced tubes}

The topologies of ellipsoidal dimples could play an important role in the thermal and hydraulic performance enhancement of the tubes [14]. A variation in the orientation of ellipsoidal dimple can offer ways of controlling heat transfer rate by altering the flow characteristics. However, according to authors' knowledge, none of the previous studies have performed a systematic investigation of thermal hydraulic characteristics of ellipsoidal dimple tubes, based on the orientations of the dimples. Therefore, this study includes all possible variations of the orientations of ellipsoidal dimples and identifies the optimum geometry based on the Performance Evaluation Criteria (PEC) [14], presented in Eqs. (14). PEC is the ratio of heat transfer enhancement to the hydraulic losses. The heat transfer enhancement is associated to the ratio of $N u$ of enhanced tube and smooth tube while the hydraulic losses are associated to the ratio of $f r$ of enhanced tube and smooth tube. Therefore, this criterion embeds both traditional indicators for the heat transfer assessment hydraulic performance estimators. 
$P E C=\frac{N u / N u_{s}}{\left(f r / f r_{s}\right)^{1 / 3}}$

The orientation of ellipsoidal dimples depends on the angles and patterns of the dimple. The angle of ellipsoidal dimple, as presented in Fig. 7, is an angle between major axis of the dimple and tube axis. From here onwards in this paper, the angle of the ellipsoidal dimple will be referred as placement angle $(\alpha)$. The placement angle of the dimples was varied from $0^{\circ}$ to $90^{\circ}$. The tubes with placement angles of $0^{\circ}$ and $90^{\circ}$ are presented in Fig. 8(a,b). Two type of dimple patterns were used in this study i.e. parallel and perpendicular as shown in Fig. $8(\mathrm{c}, \mathrm{d})$. In the parallel dimple pattern, the placement angles of dimple rows were measured opposite to flow direction. Whereas, in perpendicular pattern, the placement angles of the first dimples row were measured opposite to flow direction. In the adjacent row, the placement angles were measured in the flow direction. Therefore, in the perpendicular pattern, the circumferentially adjacent dimple had an additional $90^{\circ}$ angle. Tables 4 and 5 shows different tube parameters and configurations based on the placement angle and dimple patterns respectively.

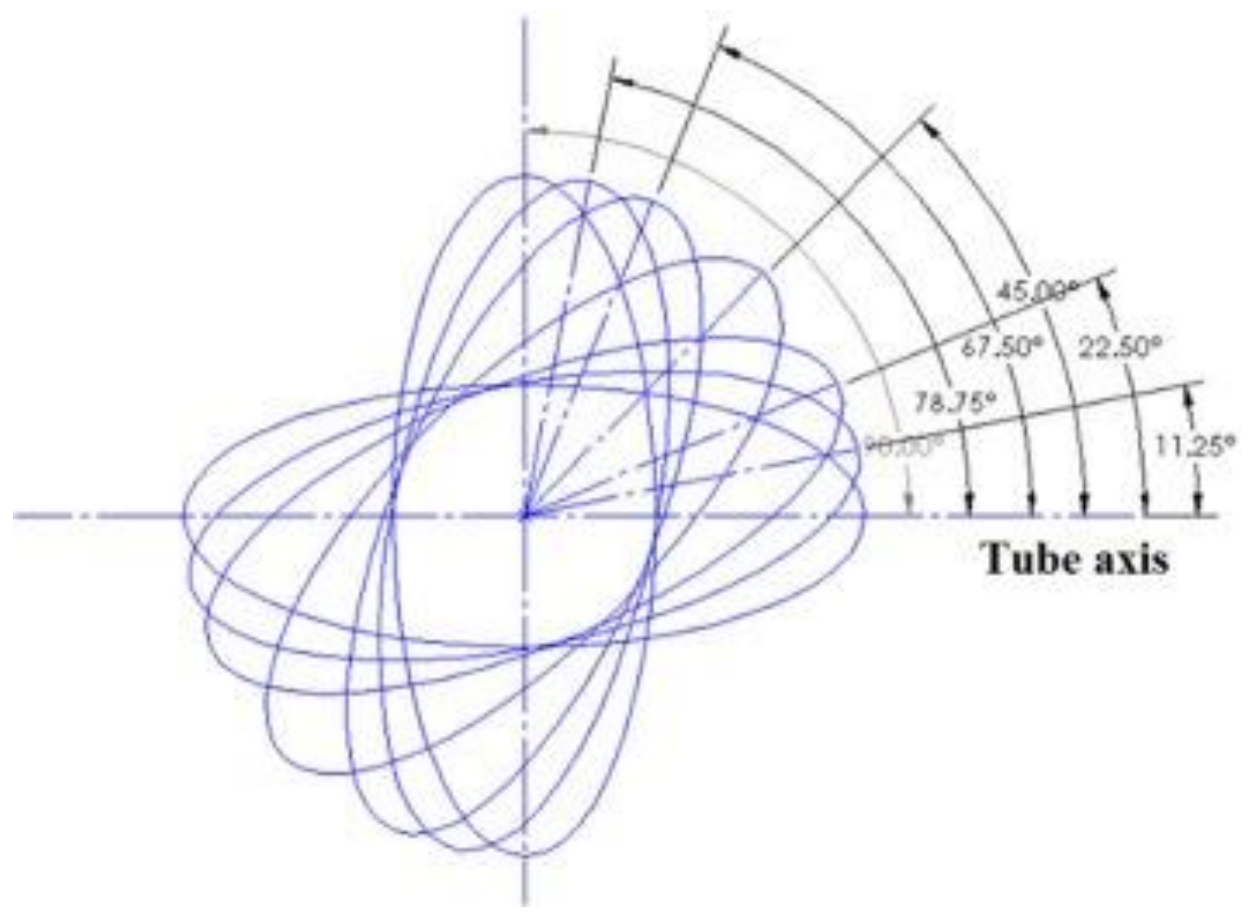

Fig. 7. Ellipsoidal placement angle. 


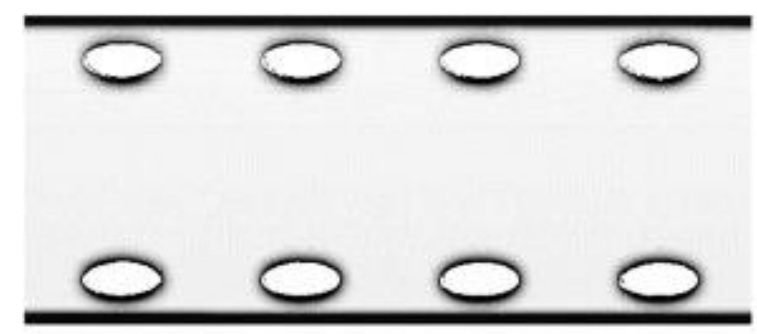

(a)

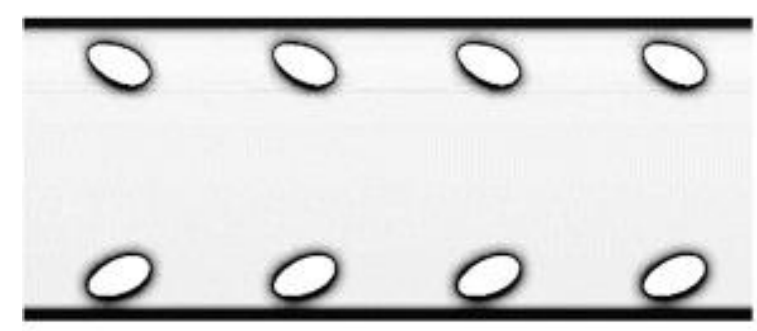

(c)

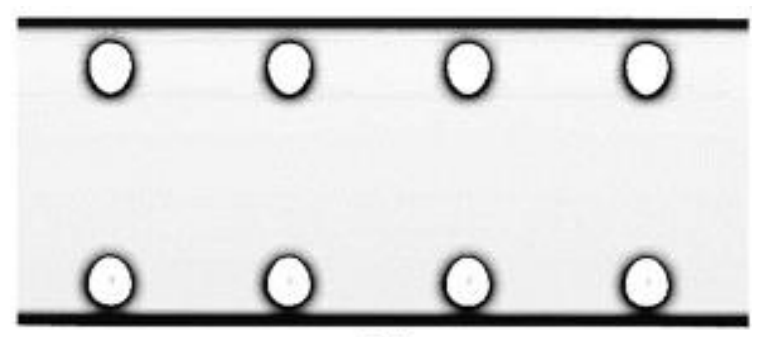

(b)

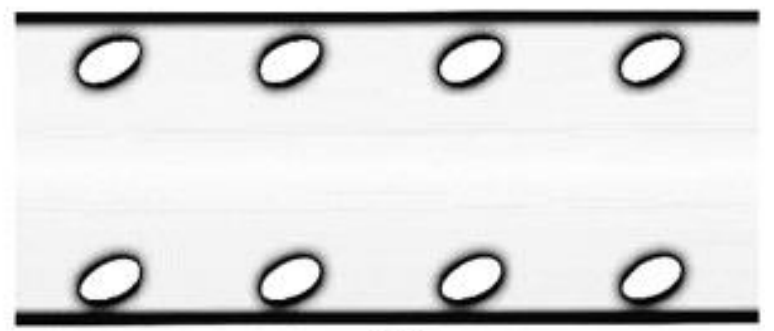

(d)

Fig. 8. Geometry of ellipsoidal dimple tubes (a) $0^{\circ}$ (tube 6), (b) $90^{\circ}$ (tube 11), (c) $45^{\circ}$ perpendicular pattern. (tube 7 ) and (d) $45^{\circ}$ parallel pattern (tube 8).

Table 4. Shape parameters of ellipsoidal dimple used in the tubes 6-15.

\begin{tabular}{llllll}
$\mathrm{D}_{\mathrm{h}}(\mathrm{mm})$ & $\mathrm{H}(\mathrm{mm})$ & $\mathbf{P}(\mathrm{mm})$ & $\mathrm{D}_{\mathbf{p}}(\mathrm{mm})$ & Star & Tube Configuration \\
\hline 17.272 & 1.1686 & 10 & 3.89506 & 4 & Inline
\end{tabular}

Table 5. Orientations of all tubes used in current study.

Sr. No Dimple placement angle $(\alpha)$

Tube 6

$0^{\circ}$

Tube 7

$45^{\circ}$ Perpendicular

Tube 8

$45^{\circ}$ Parallel

Tube 9

22.5 $5^{\circ}$ Perpendicular

Tube 10

67. $5^{\circ}$ Perpendicular

Tube 11

$90^{\circ}$

Tube 12

$22.5^{\circ}$ Parallel

Tube 13

$67.5^{\circ}$ Parallel

Tube 14

$11.25^{\circ}$ Parallel

Tube 15

$78.75^{\circ}$ Parallel 


\subsection{Average flow and heat transfer characterization using RANS}

The effects of the topologies of dimples on the thermal and hydraulic performance of the enhanced tubes are presented in Fig. 9. The ellipsoidal $45^{\circ}$ parallel dimpled tube (tube 8) showed the highest heat transfer rate in comparison to all the other tubes. The ellipsoidal $45^{\circ}$ perpendicular dimpled tube (tube 7) and Ellipsoidal $22.5^{\circ}$ parallel (tube 12) demonstrated enhanced thermal heat transfer rate in comparison to both $0^{\circ}$ and $90^{\circ}$ dimpled tubes (tube 6 and tube 11). This showed that the heat transfer rate was greatly modified by the inclination of dimpled tube. It is important to mention that the tube 6 showed lowest value for $N u$ as observed in Fig. 9(a). Interestingly, the heat transfer rate of each tube increased as the placement angles increased from $0^{\circ}$ to $45^{\circ}$ but decreased as the placement angle was increased from $45^{\circ}$ to $90^{\circ}$. Tube 7 and Tube 8 which refer to ellipsoidal $45^{\circ}$ perpendicular and parallel dimple orientations showed better thermal performance than all other tube configurations. However, the thermal performance cannot be the only performance criteria to identify the optimum configuration of the enhanced tube. Hydraulic performance of the tubes is also a crucial factor in the design of the dimpled tubes. For all the tubes, $f r$ showed a decreasing trend with respect to the Re. The relative ranking of the tubes in Fig. 9(b) resembled the ranking observed in Fig. 9(a). Ellipsoidal $45^{\circ}$ parallel tube experienced the higher-pressure loss and the ellipsoidal $0^{\circ}$ dimpled tube had lowest hydraulic loss. The $f r$ values of the rest of the tubes were between the fr values of these two limiting tubes arrangements.

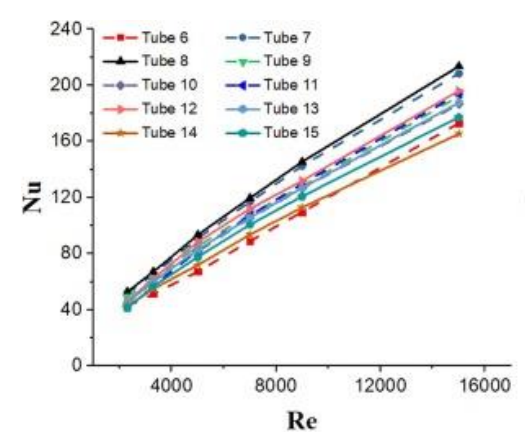

(a)

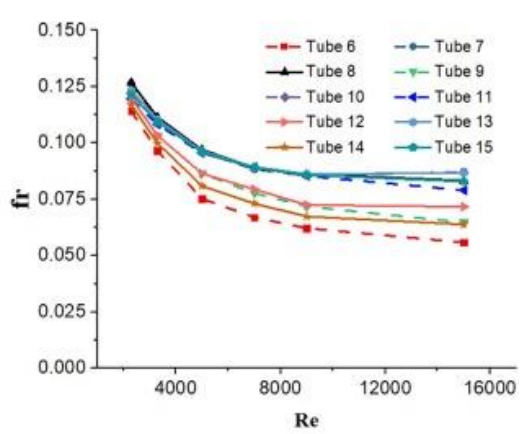

(b)

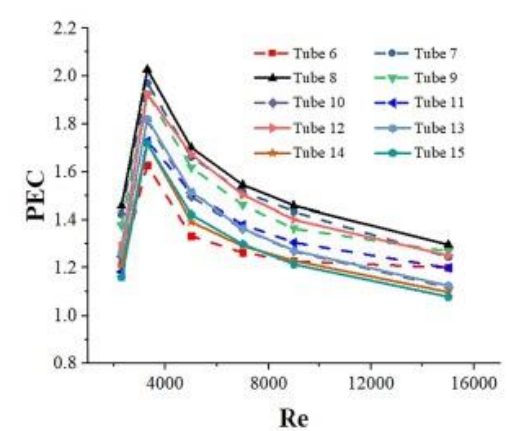

(c)

Fig. 9. Comparison between the tubes with equivalent dimples volumes (6-15), (a), (b) and (c) PEC. 
Fig. 9(c) shows the PEC of tube 6 to tube 15 for different Reynolds numbers. The results showed that initially PEC of all tubes increased for $2300 \leq R e \leq 3200$ and decreased for $R e>3200$. This trend was a result of flow transition from laminar to turbulent flow. In the pure laminar regime, flow inside the tube remained attached to the surface and therefore high friction factor values were observed. However, during the transition from laminar to turbulent flow, the friction factors reduced rapidly owing to the onset of flow detachment across the dimples. The enhanced fluid-surface interactions also increased the heat transfer in the tubes. Therefore, PEC which is simply a ratio of $N u$ and $f r$, increased rapidly in transition flow regime. However afterwards in fully turbulent regime this effect became gradually decreasing as the flow detachment resulted in persistent pressure loss in the dimpled tubes.

A significant variation in the value of $\mathrm{PEC}$ was observed as the placement angle increased from $0^{\circ}$ to $45^{\circ}$. The PEC of the $90^{\circ}$ ellipsoidal dimpled tube (tube 11) showed average performance. The tube 8 showed highest PEC value whereas tube 7 had a slightly lower PEC. Moreover, tubes parallel patterns demonstrated higher PEC than tubes with perpendicular patterns.

Thermo-hydraulic performance enhancement of $45^{\circ}$ dimpled tube in comparison to the other tubes can be associated to the flow dynamics. In this regard, Fig. 10 presents velocity contours of tube 6 , tube 8 and tube 11 in axial and circumferential directions. The velocity profile evolution was observed to be completely different in all three tubes. In Fig. 10(a), it can be observed that initially the tube 6 which was an ellipsoidal $0^{\circ}$ dimpled tube had a very thin velocity core in comparison to tube 8 (ellipsoidal $45^{\circ}$ ) and tube 11 (ellipsoidal $90^{\circ}$ ). The velocity core of the tube 11 appeared to remain constant throughout the tube. The velocity core of tube 8 comparing to tube 11 was also thinner in the beginning but it quickly grown up and become thicker than the tube 11 . The flow patterns in the velocity boundary layer of tube 6 and tube 11 showed that the flow was completely detached from the wall and the dimple surface whereas in case of tube 8 , the flow remained attached to the wall and interacted with the dimple. This could be the reason that the $f r$ of tube 8 was higher. However, this could be also due to the fact that the enhanced fluid contact with the surface enhanced the heat transfer rate. The temperature distributions are presented in Fig. 10(b) which showed that tube 8 had much thicker thermal boundary layer than the tube 6 and tube 11 . The heat transfer rate of the tube 6 was the lowest of all due to minimal fluid wall interaction. 


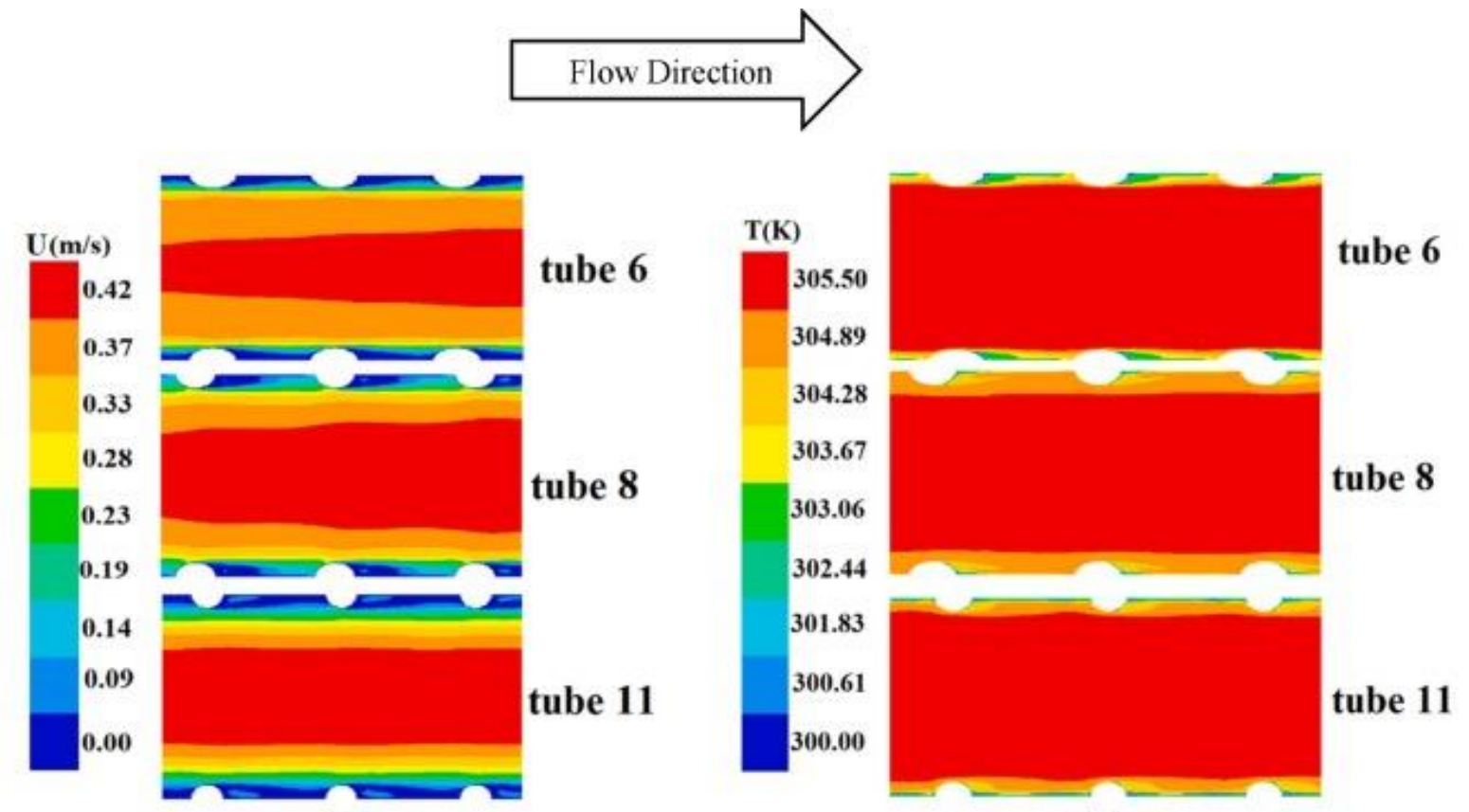

(a)

(b)

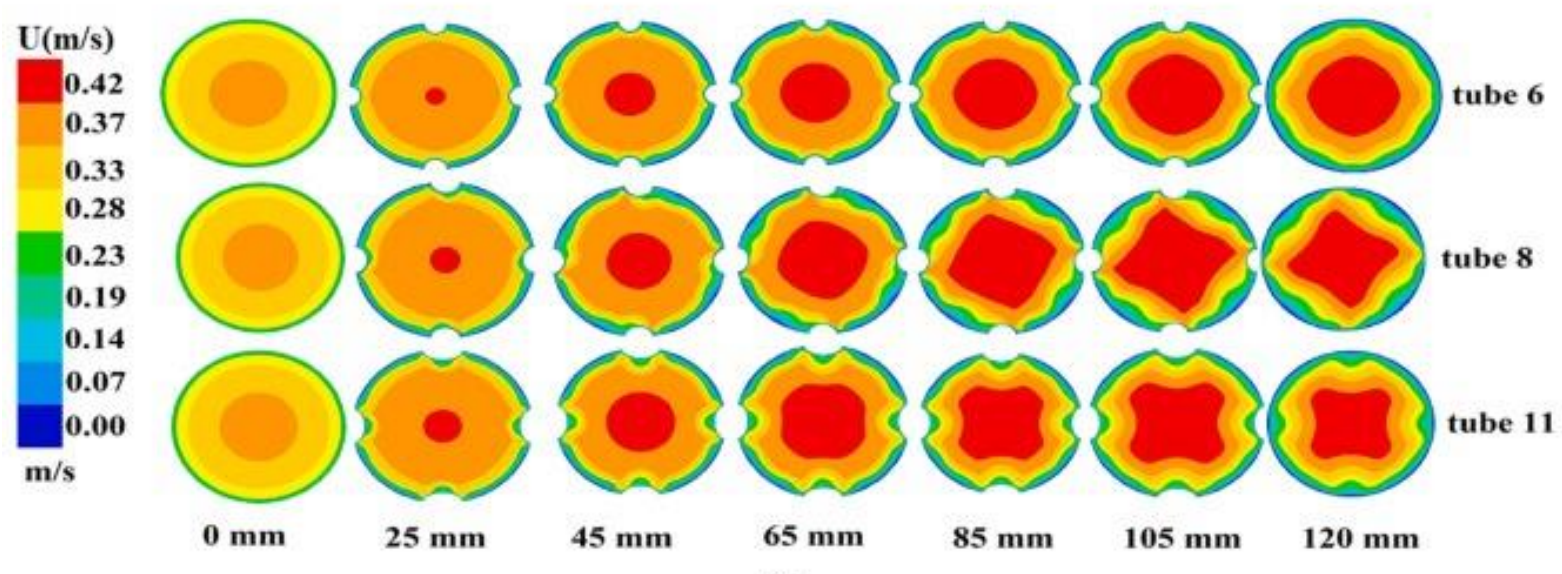

(c)

Fig. 10. Flow distribution of tubes (6, 8, and 11), (a) velocity distribution along the flow domination, (b) temperature distribution along the axis of tubes and (c) velocity distribution at different cross-sections. 
The circumferential cross-sections of velocity fields at different axial locations are presented in the Fig. 10(c) for tube 6, 8 and 11. It is interesting to note that each tube had a unique shape of the velocity core due to the different orientations of ellipsoidal dimples. The velocity core of the tube 6 was circular in shape while the tube 11 and tube 8 were square and diamond shaped respectively. The core region of the tube 8 was larger than the other two tubes. The velocity profile development affected the heat transfer rate but in order to understand the role of dimple orientation, it was necessary to observe the thermal effects more closely. For this purpose, the surface temperature and $N u$ distributions are presented, for tube 6,8 and 11, in Fig. 11(a) and (b), respectively. The temperature and Nudistribution on the surface of the tube and dimple were completely different. However, a common pattern in the flow over the dimples for tube 6 and 11 was observed which highlighted the fact that the flow detached very early from the surface of the dimples. The flow detachment forced the fluid streams of tube 6 and 11 to minimize the fluid-dimple contact of the proceeding dimples which limits the thermal performance of these tubes. However, in case of tube 8 , the fluid-dimple interaction was enhanced and therefore heat transfer was promoted. Although, it was observed that the thermal performance of the tube 8 was superior to the other tubes but the questions regarding the mechanism of heat transfer and associated flow physics remained open. Therefore, it was essential to investigate the instantaneous flow behavior of the flow over dimples.

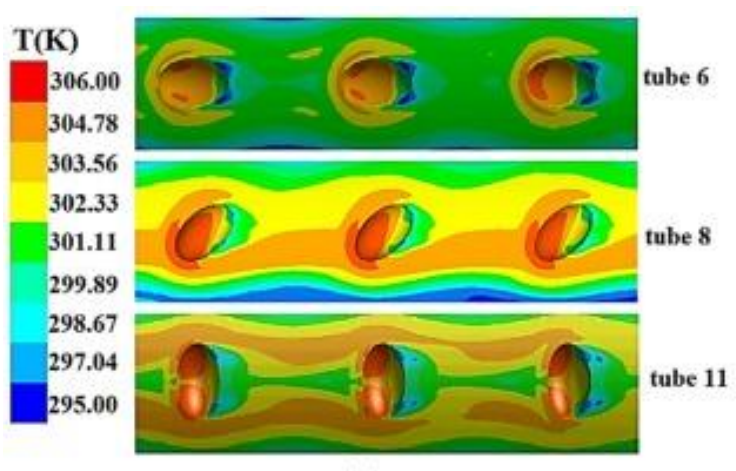

(a)

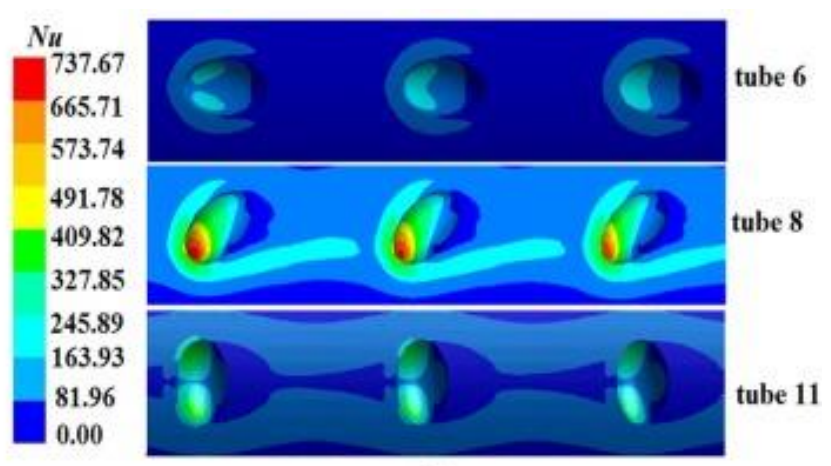

(b)

Fig. 11. Contours on the surface of tubes $(6,11$ and 8), (a) temperature and (b) Nu.

\subsection{Instantaneous flow and heat transfer characterization}

The effects of flow dynamics on the heat transfer enhancement were studied by investigating distribution of $\mathrm{Nu}$, pressure and velocity over the surface of different topologies of ellipsoidal dimples presented in Fig. 12(a to $\left.\mathrm{c}^{\prime \prime}\right)$. It is apparent from the Fig. 12 that the flow experienced a stagnation point (point A) at the leading edge of the dimples and therefore the fluid velocity reached local minima. The local was highest at the stagnation point and lowest in the wake of the dimples. After experiencing the stagnation point, the fluid accelerated in the upstream direction of the dimple due to favorable pressure gradient. The fluid velocity reached local maxima at the top and bottom of the dimple indicating symmetric flow behavior for tube 6 and tube $11\left(0^{\circ}\right.$ and $90^{\circ}$ dimples $)$. After point $\mathrm{C}$, a combined effect 
of viscous forces and adverse pressure gradient forced the flow to decelerate rapidly. The flow eventually detached at point $\mathrm{C}$ initiating unstable detached shear layer of the flow producing a large vortex in the wake of the dimple. The shear layers re-attached at point $\mathrm{E}$ for tube 6 , tube 11 , as presented in Fig. 12(a") and (c") respectively. The flow re-attachment points of both tube 6 and 11 were at the centerline of the dimples which deflected the flow streams over the top and bottom of the proceeding dimples. This in turn reduced the contact between the fluid and dimples. This flow behavior led to limited thermal enhancement of the tube 6 and tube 11 .

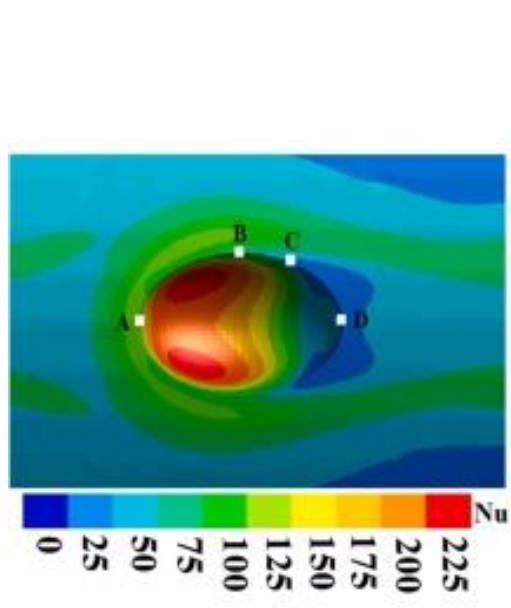

(a)

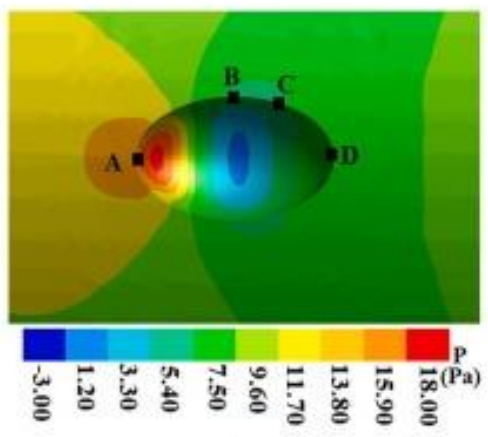

(a')

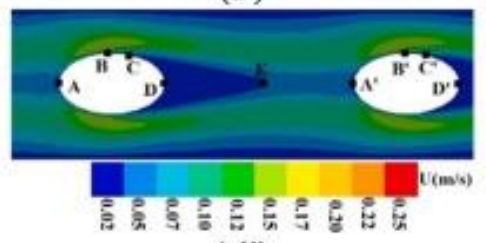

(a')

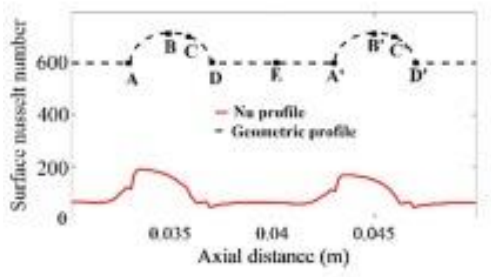

(a"')

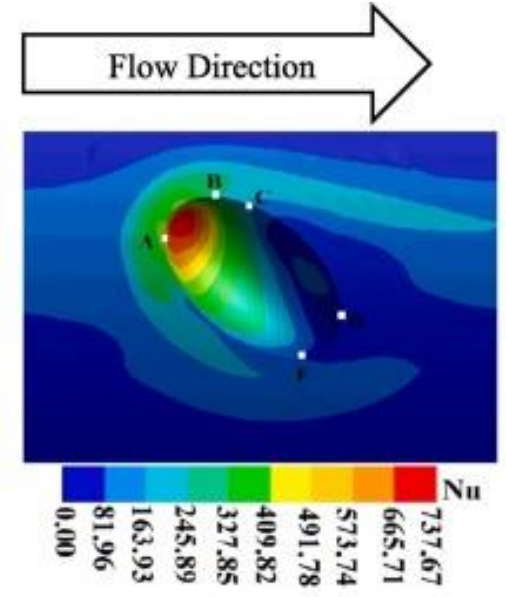

(b)

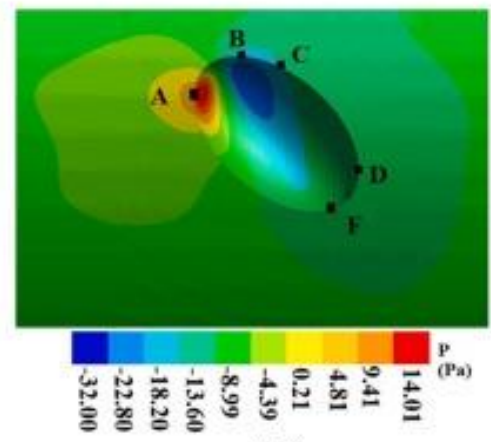

(b')

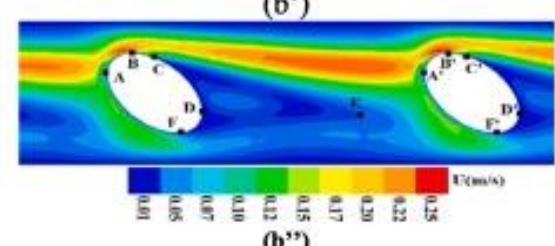

(b")

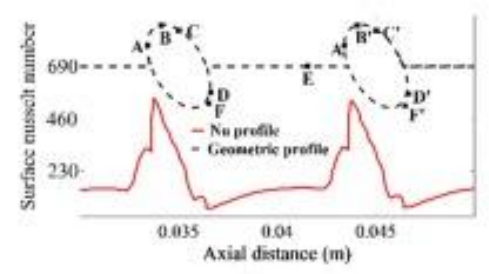

(b'"')

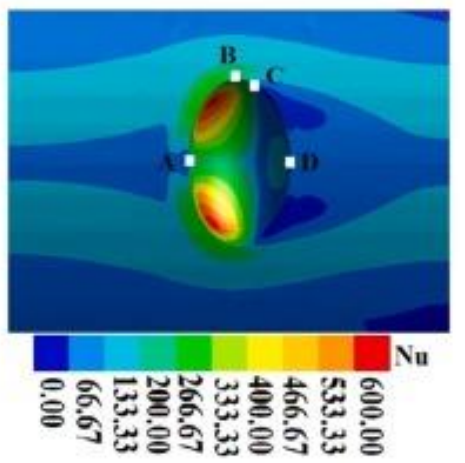

(c)

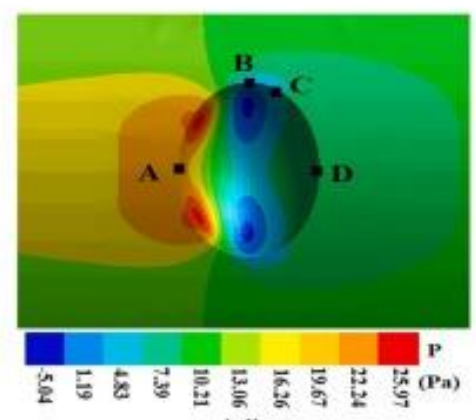

(c')

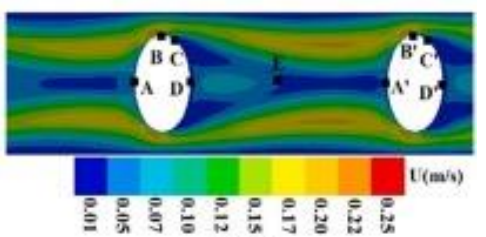

(c")

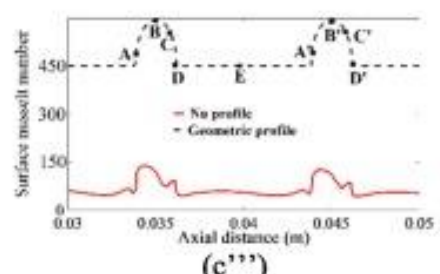

(c"')

Fig. 12. Assessment of tube 6,8 and $11(a, b, c)$ local Nu distribution over a single dimple of tubes; ( $a^{\prime}$, $\left.\mathrm{b}^{\prime}, \mathrm{c}^{\prime}\right)$ pressure distribution; $\left(\mathrm{a}^{\prime \prime}, \mathrm{b}^{\prime \prime}, \mathrm{c}^{\prime \prime}\right)$ velocity distribution at $0.0083 \mathrm{~m}$ cross-section of tubes; $\left(\mathrm{a}^{\prime \prime \prime}, \mathrm{b}^{\prime \prime \prime}\right.$, $\left.\mathrm{c}^{\prime \prime \prime}\right)$ local Nu distribution over two dimples tubes. 
The flow and the associated wake dynamics of tube $8\left(45^{\circ}\right.$ dimples $)$ were fairly different in comparison to the symmetric configurations of tube 6 and tube 11. In case of tube 8 , the flow over the inclined ellipsoidal dimple caused asymmetric pressure distribution over the dimple which yield different velocity maxima locations at the top and bottom half of the dimples comparing to tube 6 and tube 11 .

At the bottom half of the inclined dimple, the adverse pressure gradient was not as severe as found in symmetric cases due to the gradual slope of the bottom surface. This allowed the fluid to remain attached with the surface and delayed its separation at point F. On the other hand, the separation of the flow over the top half of the dimple at point $C$ occurred almost immediately at the top edge due to severe adverse pressure gradient. As a result, asymmetric flow detachment led to an asymmetric wake as shown in Fig. 12( $\left.b^{\prime}\right)$ and $\left(b^{\prime \prime}\right)$. The flow reattachment point $E$ of ellipsoidal dimple was located well below the centerline of the dimples which helped the flow to interact with frontal area of the next dimple and promotes the heat transfer. It was also confirmed by the plots of local surface along the axial direction of the tubes, at the centerline of the dimples, in Fig. 12( $\left(\mathrm{a}^{\prime \prime \prime}\right),\left(\mathrm{b}^{\prime \prime \prime}\right)$ and $\left(\mathrm{c}^{\prime \prime \prime}\right)$. The of tube 8 was significantly higher than the tube 6 and tube 11 .
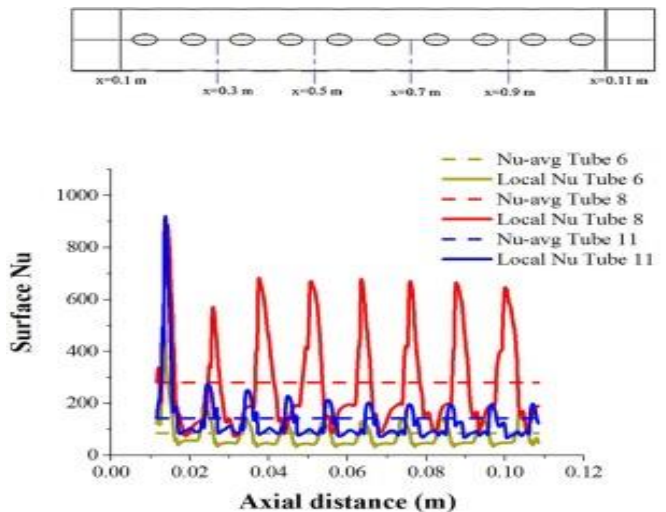

(a)

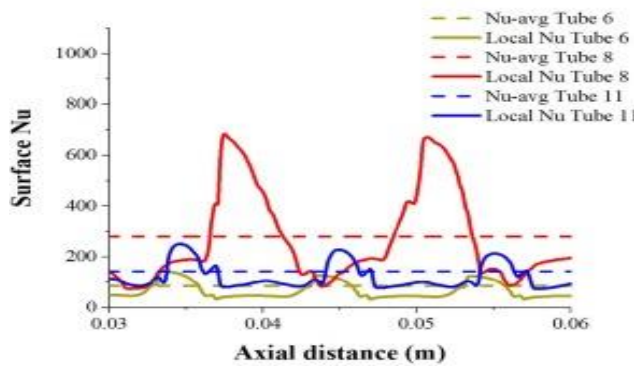

(c)

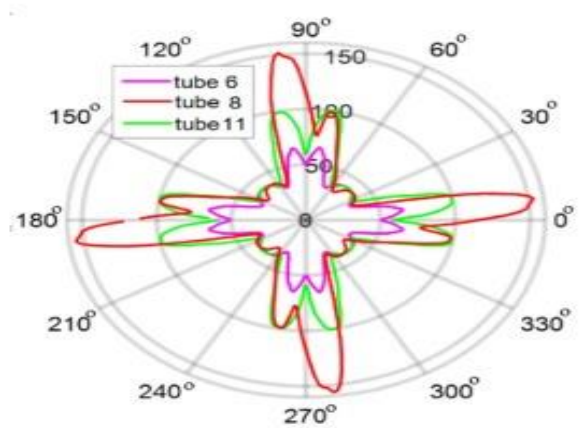

(e)
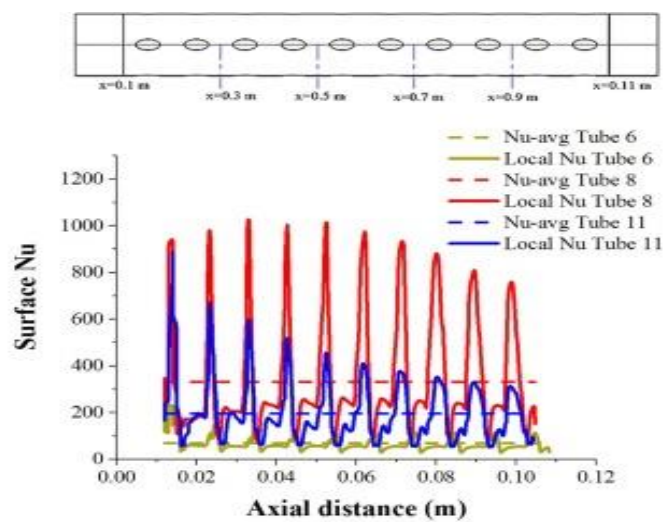

(b)

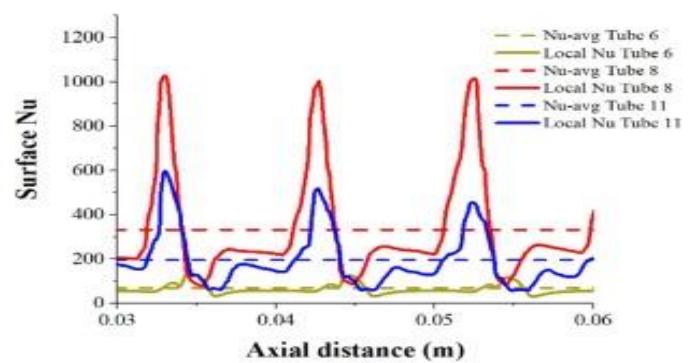

(d)

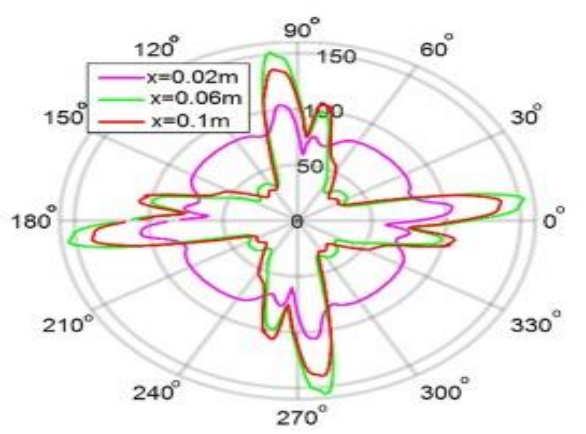

(f)

Fig. 13. Local of tubes 6,8 and 11 (a) at mid line $\mathrm{z}=0 \mathrm{~m}$ (b) at mid line $\mathrm{z}=0.001 \mathrm{~m}$ (c) zoomed section at mid line $\mathrm{z}=0 \mathrm{~m}(\mathrm{~d})$ zoomed section at mid line $\mathrm{z}=0.001 \mathrm{~m}$ (e) in circumferential direction at 0.06 $m$ in axial direction; (f) Local of tubes 8 in circumferential direction at different axial locations. 
In order to further elaborate the heat transfer enhancement, a comparison of local for complete tubes is shown in Fig. 13. The local at the mid line at and are presented in Fig. 13(a and b). The crest of local represent dimple location while the flat lines of represent the spacing between the dimples. The were comparable for all three tubes (tube 6,8 and 11) for first dimples as the flow interacted with the complete frontal area of the dimple. However, the flow separation occurred as soon as the flow crossed over the first dimple and a wake was formed at the back of the dimple. The wake at the back of the dimples dictated the flow interaction with dimples. As explained earlier, the flow was deflected over the proceeding dimples by the symmetric wake of the $0^{\circ}$ dimpled tube (tube 6 ) and $90^{\circ}$ dimpled tube (tube 11) which reduced the heat transfer whereas the asymmetric wake of $45^{\circ}$ dimpled tube (tube 8 ) allowed the flow to interact with the complete frontal area of the proceeding dimple and therefore enhanced the heat transfer rate. This was confirmed by the axial profiles in Fig. 13(a and b) which showed that tube 8 had much higher values than tube 6 and tube 11. The average value of Nusselt number of tube 6,8 and 11 were 85.9, 280 and 142.8 in case of mid line at $0 \mathrm{~m}$ and while 69.3,332.3 and 195.2 respectively, in Fig. 13(b) case of mid line at $0.001 \mathrm{~m}$. Tube 6 had the lowest heat transfer rate in comparison to the other tubes. For further elaboration, local at zoomed sections of tubes 6, 8 and 11 at the mid line at and were presented in Fig. 13(c and d).

The role of wake in the thermal performance was further elaborated by investigating the values at circumferential plane in between the two dimples. For this purpose, the distributions of for tubes 6 , tube 8 and tube 11 along the circumferential directions at, which correspond to a cross-sectional plane at $60 \mathrm{~mm}$ from the inlet, are presented in Fig. 13(e). The four peaks of correspond to the trailing edges of the dimples of each tube. Tube $8\left(45^{\circ}\right.$ parallel) showed higher heat transfer rate, which was 3 times higher than a smooth tube while tube 6 and 11 enhanced heat transfer rate by 1.3 and 1.96 times the smooth tube, respectively. Again, the tube 8 showed better thermal performance than other tubes.

The along the circumferential direction at different downstream locations of tube 8 are presented in Fig. 13(f). The initially increased in the downstream direction but decreased near the tube outlet. At 20 $\mathrm{mm}$ downstream position, the was two times higher than the of smooth tube and further increased to 3 times at. At the tube exit, was 2.7 times the due to the unsteadiness in the flow field at the tube exit.

\subsection{Transient flow and heat transfer characterization using Large Eddy Simulation}

The effect of orientation of ellipsoidal changed the flow patterns significantly. Therefore, strong influence of flow dynamics was involved in the heat transfer enhancement. The associated wake dynamics and fluctuating flow were expectedly transient phenomena. For this purpose, Large Eddy Simulation (LES) was performed which resolved integral and inertial sub-range scale eddies in the flow and modeled the Kolmogorov scale eddies. Since, LES was computationally very expensive, therefore it was used to study only tube 8 and tube 11 at with three dimples in axial direction. A structured mesh with 4.4 million computational cells was used with wall . A fixed time step of $50 \mu$ s was employed for both cases for a flow time duration of $300 \mathrm{~ms}$. Temporal distributions of velocity of tube 8 and tube 11 are presented in Fig. 14(a) and (b) respectively. Initially at $50 \mathrm{~ms}$, a vortex pair emerged in the wake of the dimple for tube 11. This vortex travelled in the downstream direction and eventually interacted with the next dimple. At $100 \mathrm{~ms}$, flow paths were created over the top and bottom half of the dimples. After $150 \mathrm{~ms}$, the tail of the wake of first dimple attached with the stagnation point of the downstream dimples which forced the fluid streams to travel over the edges of the dimples. It was more elaborated in the streamlines that wake of the tube 11 was symmetric and thick which did not allow the flow to interact with the downstream dimple. Furthermore, in tube 11as observed in Fig. 14(b), the vortex length of second dimple increased and eventually resulted in vortex shedding. The vortex shedding between the dimpled caused additional flow deflection and further reduction of heat transfer in tube 11. 


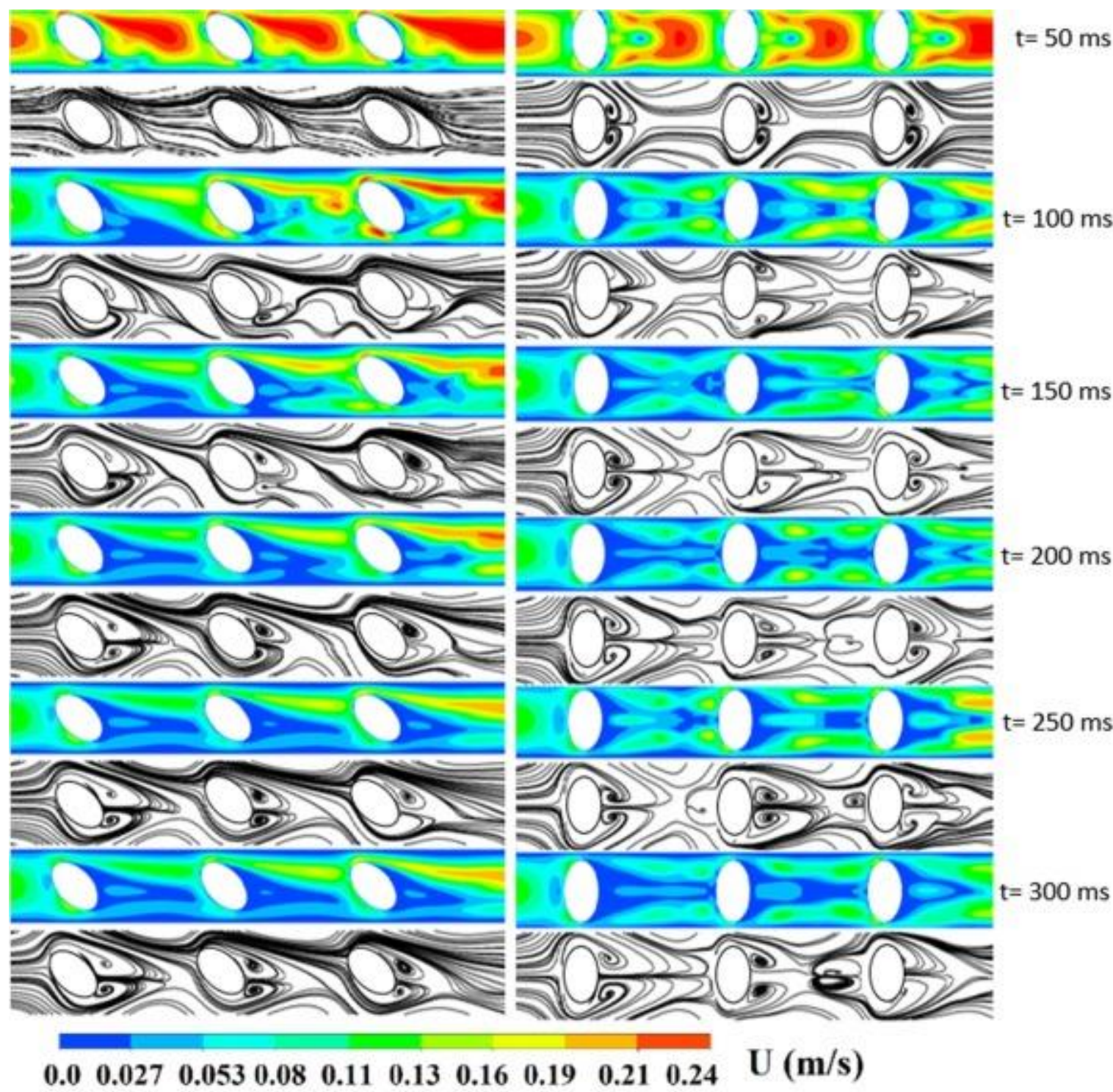

(a)

(b)

Fig. 14. Velocity contours and streamline plots from LES results of (a) tube 8 (b) tube 11.

However, the wake pattern of tube 8 was different from the tube 11. The Fig. 14(a) shows that the wake behind the dimples emerged from the bottom half of the dimples which slowly moved upwards and attached to the bottom half of the downstream dimple. The asymmetric wake allowed the flow to interact with the downstream dimples and produced a narrow flow path at the bottom half of the dimple. This can also be observed in streamlines which showed that the fluid-dimple interaction was enhanced which in turn increased the heat transfer rate. 


\section{Proposed correlations for different ellipsoidal dimple topologies}

The correlations of $\mathrm{Nu}$ and $f r$, for all possible topologies of ellipsoidal dimples, have been proposed in Eqs. (15), (16), respectively. The relationships of $N u$ and $f r$ were defined as a function of Re and placement angle $\alpha$. The Reynolds number can be varied between $2300 \leq R e \leq 15000$ while placement angle can be varied between $0^{\circ} \leq \alpha \leq 90^{\circ}$. The constants $a$ and $b$ of both correlations were linear functions of the placement angle $\alpha$. The comparisons of $N u$ and fr from numerical results and proposed correlations have been presented in Fig. 15(a) and (b), respectively. A good agreement between the correlation and numerical results was observed. The maximum difference for $\mathrm{Nu}$ remained below $20 \%$ and below $25 \%$ in case of $f r$, for all placement angles and Reynolds numbers.

$$
\begin{aligned}
& N u=a R e^{b(1+0.3 \alpha / 2 \pi)} \quad\left\{\begin{array}{c}
a=-0.0006617 \alpha+0.13474 \\
b=0.00051111 \alpha+0.7412
\end{array}\right\} \\
& f r=\left[a \cdot \ln \left(\operatorname{Re} \varphi \frac{\alpha}{2 \pi}\right)+b\right]^{-3 / 2} \quad\left\{\begin{array}{c}
a=-0.0079747 \alpha+1.2462 \\
b=0.059878 \alpha-5.3636
\end{array}\right\}
\end{aligned}
$$

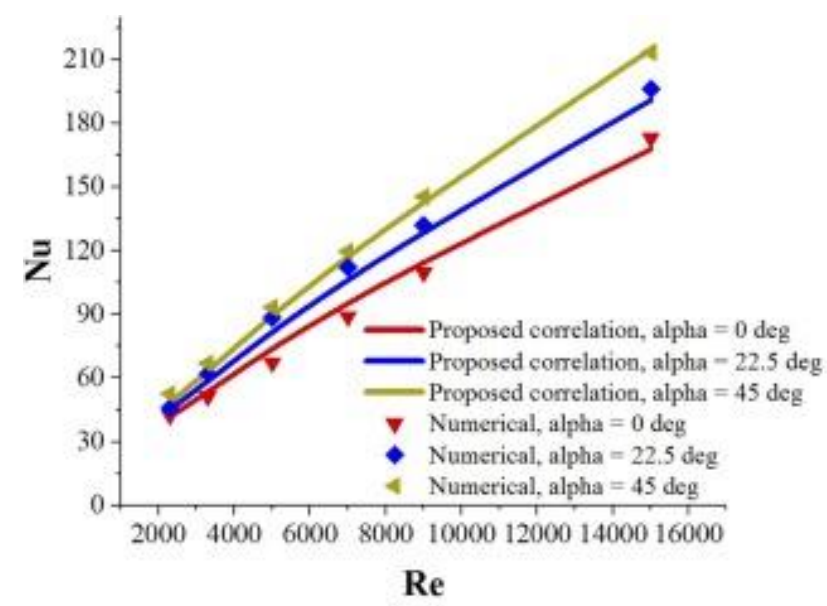

(a)

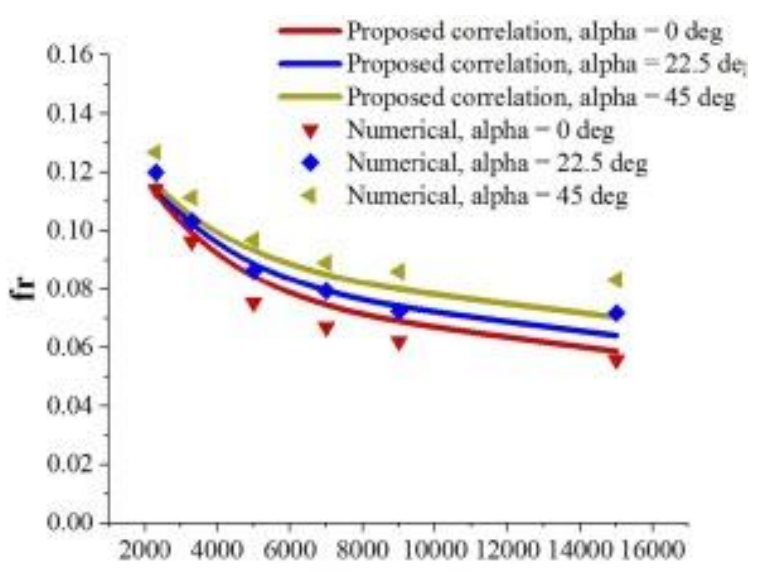

$\operatorname{Re}$

(b)

Fig. 15. Comparison of numerical and correlation results (a) Nuand (b) f 


\section{Conclusion}

Thermal hydraulic performance of flow through externally heated enhanced tubes with constant surface heat flux was investigated. The tubes were enhanced by introducing different topologies of ellipsoidal dimples of equivalent volumes. It was observed that in general, in comparison to smooth tubes, all configurations of ellipsoidal dimpled tubes demonstrated improved overall performance of the enhanced tubes. However, the $45^{\circ}$ ellipsoidal dimpled tube (tube 8) enhanced the average heat transfer rate, average thermo-hydraulic performance by $119.5 \%$ and $58.1 \%$ in comparison to the smooth tube, respectively. At low $R e$, tube 8 achieved the average surface $\mathrm{Nu}$ of 52.7 which was $112 \%$ higher than that of smooth tube and $25 \%$ higher than $\mathrm{Nu}$ of tube 6 and tube 11 . At high $R e$, the average surface $N u$ of tube 8 increased to 213.5 which was $85 \%$ higher than $N u$ of smooth tube while $23 \%$ and $10 \%$ higher than tube 6 and tube 11 , respectively. The dimple fluid interaction also increased the friction factor of the tube however thermo-hydraulic performance of tube 8 was much higher than that of smooth tubes. The performance evaluation criteria of tube 8 varied between 1.45 and 1.30 for $2300 \leq R e \leq 15000$. The better performance of tube 8 observed in the study is observed to be linked to asymmetric wake behind the dimples which provided a path for the flow to interact with the succeeding dimples. The increased interaction between the flow and dimples promoted heat transfer. Whereas, tube 6 and tube 11 decreased the flow interaction with dimples by deflecting the flow streams over the dimples which subsequently reduced the heat transfer. In the end, correlations for $\mathrm{Nu}$ and $f r$ were proposed as a function of Re and dimple placement angle $\alpha$ which covered each configuration of the ellipsoidal dimpled tube. The for $N u$ and $f r$ could readily be obtained for different topologies of ellipsoidal dimpled tube with reasonable accuracy for $2300 \leq R e \leq 15000$.

\section{Declaration of Competing Interest}

The authors declare that they have no known competing financial interests or personal relationships that could have appeared to influence the work reported in this paper. 